\title{
Fractal Reconstruction of Sub-Grid Scales for Large Eddy Simulation
}

\author{
Emmanuel O. Akinlabi ${ }^{1}$ (D) . Marta Wacławczyk ${ }^{1}$. Szymon P. Malinowski ${ }^{1}$. \\ Juan Pedro Mellado ${ }^{2}$
}

Received: 16 November 2018 / Accepted: 2 May 2019 / Published online: 28 May 2019

(C) The Author(s) 2019

\begin{abstract}
In this work, the reconstruction of sub-grid scales in large eddy simulation (LES) of turbulent flows in stratocumulus clouds is addressed. The approach is based on the fractality assumption of turbulent velocity field. The fractal model reconstructs sub-grid velocity field from known filtered values on LES grid, by means of fractal interpolation, proposed by Scotti and Meneveau (Physica D 127, 198-232 1999). The characteristics of the reconstructed signal depend on the stretching parameter $d$, which is related to the fractal dimension of the signal. In many previous studies, the stretching parameter values were assumed to be constant in space and time. To improve the fractal interpolation approach, we account for the stretching parameter variability. The local stretching parameter is calculated from direct numerical simulation (DNS) data with an algorithm proposed by Mazel and Hayes (IEEE Trans. Signal Process 40(7), 1724-1734, 1992), and its probability density function (PDF) is determined. It is found that the PDFs of $d$ have a universal form when the velocity field is filtered to wave-numbers within the inertial range. In order to investigate Reynolds number ( $R e$ ) dependence, we compare the inertial-range PDFs of $d$ in DNS and large eddy simulation (LES) of stratocumulus cloud-top and experimental airborne data from Physics of Stratocumulus Top (POST) research campaign. Next, fractal reconstruction of the subgrid velocity is performed and energy spectra and statistics of velocity increments are compared with DNS data. It is assumed that the stretching parameter $d$ is a random variable with the prescribed PDF. We show that the agreement with the DNS is in such case better and the error in mass conservation is smaller compared to the use of constant values of $d$. The motivation of this study is to reproduce effect of sub-grid scales on a motion of Lagrangian particles (e.g. droplets) in clouds.
\end{abstract}

Keywords Fractal interpolation technique · Turbulence · Large Eddy simulation · Sub-grid scale model $\cdot$ Lagrangian particles

Emmanuel O. Akinlabi

emmanuel.akinlabi@fuw.edu.pl

1 Institute of Geophysics, Faculty of Physics, University of Warsaw, Warsaw, Poland

2 Max-Planck Institute for Meteorology, Hamburg, Germany 


\section{Introduction}

Lagrangian tracking of particles in turbulent flows seen in nature requires huge computational resources because the flow of a carrier fluid is spatially and temporally complex, with structures in a wide range of scales. The ratio between the largest and smallest scales of turbulence $(\operatorname{Re} \approx L / \eta)$ observed in such flows can be as high as $10^{9}$. The most detailed research tool, which can resolve all turbulent scales and accurately predict particle trajectories, is the DNS [1]. Owing to its unrealistic computational cost at high Reynolds numbers, LES is used as a compromise to calculate the large scale features (resolved) of the flow while sub-grid (unresolved) scales are modelled. Lack of sub-grid scales or sub-grid scale model errors will lead to the progressive divergence of particle trajectories when compared with those obtained in experiment or DNS [2,3]. As a result, particle statistics such as preferential concentration, average settling velocity and relative velocities are either over- or under-estimated [4-10].

Several attempts have been made to develop models for the effects of sub-grid scales of turbulence on the prediction of the Lagrangian dispersion of inertial particles. As classified by [3], there are stochastic and structural models. Stochastic models are based on the solutions of the Langevin equations [11-14] supplemented with a stochastic Wiener process term $[15,16]$. Various works on stochastic models show that they perform well at small Stokes number but have strong sentivitity to the filter size [3, 17].

Structural sub-grid models are an alternative to the stochastic models and they aim at mimicking (some of) the subgrid scales in a statistical sense. These models allow for the approximate reconstruction of two-point particle statistics at the subgrid scale [8, 9, 18]. Example of structural models are the fractal interpolation [2, 19, 20], approximate deconvolution (ADM) [21-24], spectrally optimized interpolation [25], the kinematic simulations based on Fourier modes [26-29] and low-order dynamical systems [68]. A good structural sub-grid model should not only be able to capture the statistics of small scales but also be computationally efficient and easy to use. Fractal interpolation technique (FIT) seems promising (especially for high $R e$ geophysical applications) with respect to these features. FIT was introduced to construct synthetic, fractal subgrid-scale fields applied to LES of both stationary and freely decaying isotropic turbulence [20]. The underlying assumption of the model is the existence of fractal-scale similarity of velocity fields. An attribute of the constructed sub-grid velocity depends on the stretching parameter $d$, which is related to the fractal dimension of the signal. In Scotti and Meneveau [20], it was assumed that $d$ is constant in space and time for homogeneous and isotropic turbulence. Basu et al. [30] proposed an extension of this work by developing a multiaffine fractal interpolation scheme with bi-valued stretching parameter and showed that it preserves the higher-order structure functions and the non-Gaussianity of the PDF of velocity increments. They performed extensive analyses on atmospheric boundary layer data and argued that the multiaffine closure model should give satisfactory performance in LES. Marchioli et al. [2] used fractal interpolation and approximate deconvolution technique to model sub-grid scale turbulence effects on particle dynamics in wall-bounded turbulence. They showed that FIT appears to be inefficient in reintroducing the fluid velocity fluctuation when a constant stretching parameter is assumed [31]. They concluded that an accurate sub-grid model for particles may require information on the higher-order moments of the velocity fluctuation [2].

Characteristic features of atmospheric turbulence are the inhomogeneity due to buoyancy and the presence of internal and external intermittency. Internal intermittency means that large velocity gradients are present at small scales and the PDF of velocity differences at high Reynolds number are stretched-exponential at small scales [32]. External intermittency 
refers to the co-existence of both laminar and turbulent regions in the flow. These attributes make it difficult to synthesize sub-grid velocity field using fractal interpolation since the local stretching parameters have been shown to change randomly in space and time [2, 33].

The aim of this work is to develop an improvement to FIT, which can be used as a closure model for Lagrangian tracking of particles in moderate or high Reynold number (complex) turbulent flows. We proceed by accounting for the spatial variability of the stretching parameter: first, its local value is computed with a method proposed by Mazel and Hayes [34]. Then, the PDF of local stretching parameter is used to construct subgrid velocity, starting with the filtered DNS or LES. Three examples of complex turbulent flows are investigated, first moderate Reynolds number DNS velocity field of stratocumulus-top boundary layer (STBL) [35], second, LES of stratocumulus cloud at $R e$ comparable with atmospheric turbulence [36] and, finally, experimental data from POST airborne measurements in stratocumulus clouds [37-39].

The extracted data of PDF of $d$ are used to perform $3 D$ reconstruction of subgrid eddies. Performance of the new approach is compared with FIT with the constant stretching parameter values from Ref. [20] and the multiaffine fractal interpolation scheme [30]. We calculate energy spectra of the reconstructed velocity field and the statistics of velocity increments. The focus on the statistics of velocity increments is motivated by their ability to quantify internal intermittency of small scale turbulence [32, 40, 41], which can influence inertial particle statistics in turbulent flows [40, 42, 43]. We found the new approach with random $d$ the most favourable in terms of the investigated statistics. It reproduces the Kolmogorov's $-5 / 3$ scaling of turbulent kinetic energy spectra in the inertial range with the smallest error and without spurious modulations. Moreover, PDFs of increments of the reconstructed velocity have non-Gaussian, stretched-exponential tails.

This paper is structured as follows: Section 2 describes the FIT and method for stretching parameter estimation from 1-D velocity series. Section 3 discusses briefly the DNS, LES and POST airborne datasets of stratocumulus cloud [36, 39, 44]. Section 4 compares the PDF of local stretching parameter for the three datasets. In Section 5, FIT is applied to 1-D filtered DNS velocity field. The PDF of velocity increments of the reconstructed field and PDF of DNS velocity increments are compared. In Section 5 the application of the improved FIT to POST airborne measurement and 3-D LES velocity field is also presented.

\section{Fractal Interpolation Techniques}

\subsection{Basics}

The fractal interpolation technique is an iterative affine mapping procedure to construct the synthetic (unknown) small-scale eddies of the velocity field $\mathbf{u}(\mathbf{x}, t)$ from the knowledge of a filtered or coarse-grained field $\tilde{\mathbf{u}}(\mathbf{x}, t)$ [20]. For clarity, if $\overline{\tilde{\mathbf{u}}}(\mathbf{x}, t)$ have resolution $\Delta^{\prime}$ while $\tilde{\mathbf{u}}(\mathbf{x}, t)$ have $\Delta$ such that $\Delta^{\prime}>\Delta$, the mapping operator $W[$ [.] applied to $\overline{\tilde{\mathbf{u}}}(\mathbf{x}, t)$ gives $\tilde{\mathbf{u}}(\mathbf{x}, t)$ $=W[\overline{\tilde{\mathbf{u}}}(\mathbf{x}, t)]$. To generate synthetic small scale velocity fields, the mapping is performed many times i.e. a fractal synthetic field

$$
\mathbf{u}_{f}(\mathbf{x}, t)=\lim _{n \rightarrow \infty} W^{(n)}[\tilde{\mathbf{u}}(\mathbf{x}, t)] \equiv \lim _{n \rightarrow \infty} W[W[W \ldots W[\tilde{\mathbf{u}}(\mathbf{x}, t)] \ldots]]
$$

For example, if we consider three interpolating points $\left\{\left(x_{i}, \tilde{u_{i}}\right), i=0,1,2\right\}$, the fractal interpolation reconstructs a signal $w_{j}, j=1,2$ at two additional points placed between 
points 0 and 1 and points 1 and 2, see Fig. 1 . Here, $w_{j}$ has the following transformation structure:

with constraints

$$
w_{j}\left(\begin{array}{l}
x \\
u
\end{array}\right)=\left[\begin{array}{ll}
a_{j} & 0 \\
c_{j} & d_{j}
\end{array}\right]\left(\begin{array}{l}
x \\
u
\end{array}\right)+\left(\begin{array}{l}
e_{j} \\
f_{j}
\end{array}\right), \quad j=1,2
$$

$$
w_{j}\left(\begin{array}{c}
x_{0} \\
\tilde{u_{0}}
\end{array}\right)=\left(\begin{array}{c}
x_{j-1} \\
\tilde{u}_{j-1}
\end{array}\right) \quad \text { and } \quad w_{j}\left(\begin{array}{c}
x_{2} \\
\tilde{u}_{2}
\end{array}\right)=\left(\begin{array}{c}
x_{j} \\
\tilde{u}_{j}
\end{array}\right), \quad j=1,2
$$

The parameters $a_{j}, c_{j}, e_{j}$ and $f_{j}$ can be written in terms of $d_{j}$ (called the stretching parameter) and the interpolation points $\left\{\left(x_{i}, \tilde{u_{i}}\right), i=0,1,2\right\}$. Values of $d_{j}$ fix the vertical stretching of the left and right segments at each iteration and determine characteristics of the reconstructed signal. Their values are independent of the interpolation points.

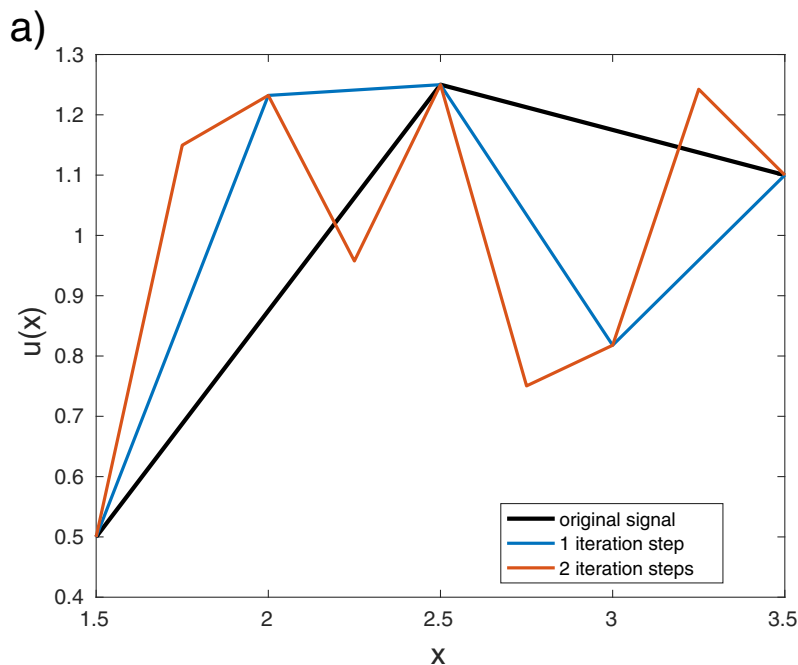

b)

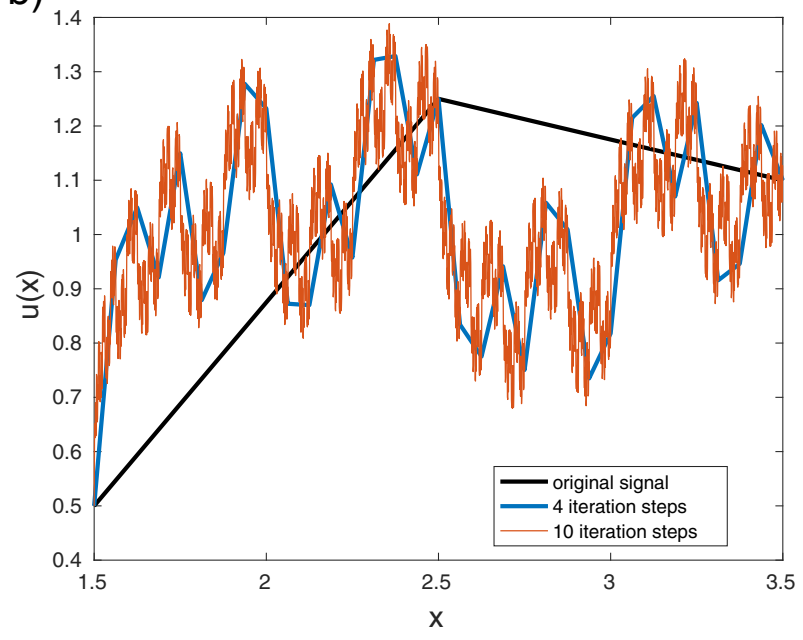

Fig. 1 Different stages during the construction of a fractal function with stretching parameter $d= \pm 2^{-1 / 3}$ after $\mathbf{a} 0,1$ and 2 reconstruction steps $\mathbf{b} 0,4$ and 10 reconstruction steps 
The iterative procedure in the limit $n \rightarrow \infty$ creates a continuous function $\mathbf{u}_{f}(\mathbf{x})$ provided that the stretching parameter $d_{j}$ obeys $0 \leq\left|d_{j}\right|<1$. Also, if $\left|d_{1}\right|+\left|d_{2}\right|>1$ and $\left(x_{i}, \tilde{u_{i}}\right)$, are not collinear, then the fractal dimension $D$ of the reconstructed signal is the unique real solution of $\left|d_{1}\right| a_{1}^{D-1}+\left|d_{2}\right| a_{2}^{D-1}=1$ (for proof, see [45]). Thus, once the stretching parameter is chosen, the remaining parameters $a_{j}, c_{j}, e_{j}$ and $f_{j}$ are given as

$$
\begin{gathered}
a_{j}=\frac{x_{j}-x_{j-1}}{x_{2}-x_{0}} \\
e_{j}=\frac{x_{2} x_{j}-x_{0} x_{j}}{x_{2}-x_{0}} \\
c_{j}=\frac{\tilde{u_{j}}-\tilde{u}_{j-1}}{x_{2}-x_{0}}-d_{j} \frac{\tilde{u_{2}}-\tilde{u_{0}}}{x_{2}-x_{0}} \\
f_{j}=\frac{x_{2} \tilde{u}_{j-1}-x_{0} \tilde{u}_{j}}{x_{2}-x_{0}}-d_{j} \frac{x_{2} \tilde{u_{0}}-x_{0} \tilde{u_{2}}}{x_{2}-x_{0}}
\end{gathered}
$$

Given that

$$
1<\sum_{j=1}^{N}\left|d_{j}\right|<2,
$$

where $N+1=N_{A}$ and $N_{A}$ is the number of anchor points (here, $N=2$ ), the stretching parameter $d_{j}$ relates to the fractal dimension $D$ of a velocity field as:

$$
D=1+\log _{N} \sum_{j=1}^{N}\left|d_{j}\right|
$$

(for proof, see [19, 45]). It is important to note that the relation between the fractal dimension and the inertial-range self-similarity of turbulent velocity field is not trivial. Orey [46] proved that almost all realizations of a Gaussian random field with a power-law spectrum, $E(k) \sim k^{-\alpha}$ with $1<\alpha<3$, give fractal dimension $D=(5-\alpha) / 2$. For Kolmogorov spectrum, $\alpha=5 / 3$, which results in $D=1.67$. Although turbulent velocity fluctuations are not Gaussian, the high-Reynolds experimental results of Praskovsky et al. [47] and Scotti et al. [48] agree with Orey's theorem. They concluded that turbulent velocity fluctuations gives a fractal dimension $D \simeq 1.7 \pm 0.05$, which is close to $D=1.67$ expected for Gaussian signals.

If a fractal dimension of $5 / 3$ relating to $-5 / 3$ Kolmogorov scaling in the inertial-range of velocity fields is assumed, then $d_{j}= \pm 2^{-1 / 3}$ (if $d_{j}$ is assumed to be the same for all grid spacings) [20]. Figure 1 shows the 1-D construction of $w_{j}$. We start from a field with three grid points and we successively apply the map $w_{j}$ with stretching parameter $d_{j}= \pm 2^{-1 / 3}$. Shown are the initial field, first, second, fourth and the tenth application of the map $w_{j}$. The energy spectrum after ten reconstruction steps is shown in Fig. 2.

In 3-D, FIT is performed separately along $x$-, $y$ - and $z$-directions, see [20]. Let us assume that the filtered field has $N$ grid points in all three directions that is, we have $\tilde{\mathbf{u}}_{i j k}$ for $i, j, k=0,1,2, \ldots, N-1$. First, 1-D intersections of the coarse-grained field $\tilde{\mathbf{u}}_{i j k}$ along $x$ - direction are created and FIT is performed for each 1-D intersection (see the blue lines on the schematic Fig. 3), that is, for each $j, k=0,1, \ldots, N-1$. We obtain a field which we denote by $\tilde{\mathbf{u}}_{i j k}^{x}$, where $i=0,1, \ldots, 2 N-1, j, k=0,1, \ldots, N-1$. Then, FIT for each 1-D intersection of $\tilde{\mathbf{u}}_{i j k}^{x}$ along $y$-direction is performed, which gives a field $\tilde{\mathbf{u}}_{i j k}^{x, y}$, where $i, j=0,1, \ldots, 2 N-1, k=0,1, \ldots, N-1$ (red lines in Fig. 3). Lastly, FIT is performed on $\tilde{\mathbf{u}}_{i j k}^{x, y}$ along $z$-direction similar to how it was performed in $x$-and $y$-directions. With this, the field $\mathbf{u}_{i j k}$, where $i, j, k=0,1, \ldots, 2 N-1$ is finally obtained. 


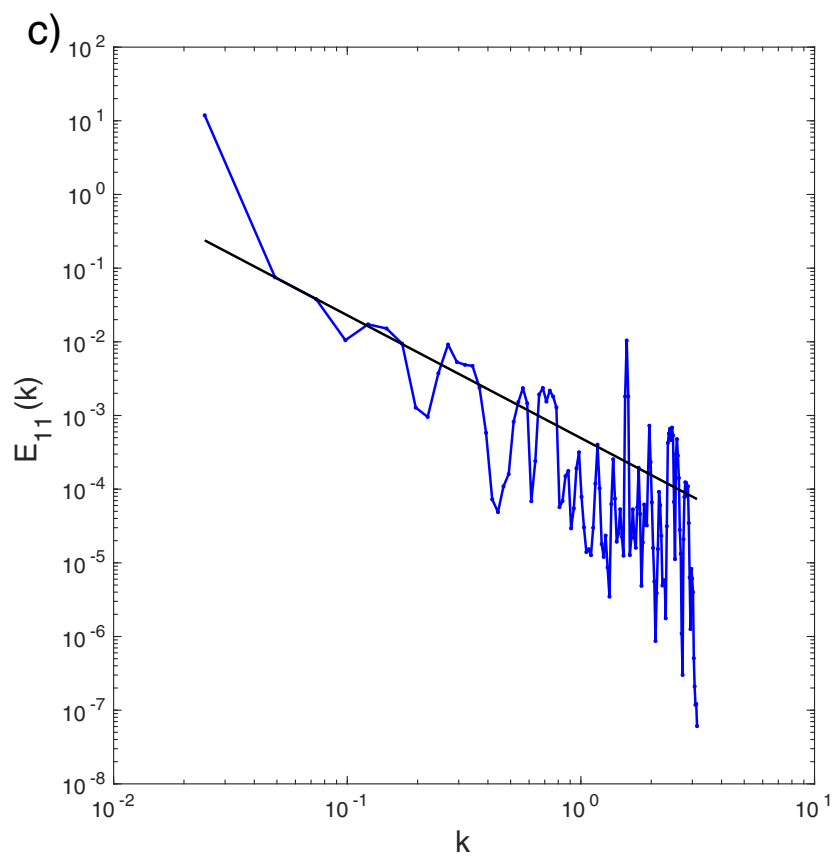

Fig. 2 Energy spectrum of the reconstructed signal after 10 iterations showing $-5 / 3$ slope

\subsection{Stretching parameter estimation}

Mazel and Hayes [34] proposed an algorithm for computing the local stretching parameter $d_{i}$ of any given arbitrary dataset. The algorithm is based on the property that the resulting fractal field is self-similar. For illustration, if we consider a dataset with 5 interpolation

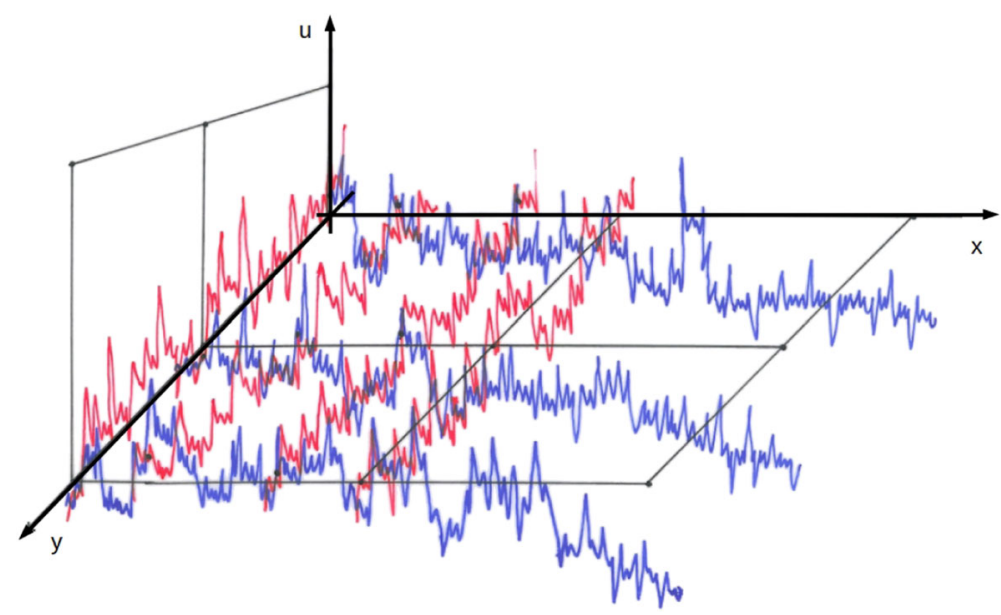

Fig. 3 Schematic picture of a process of the fractal reconstruction in $2 \mathrm{D}$ 
points $\left\{\left(x_{i}, u_{i}\right), i=0,1,2,3,4\right\}$, let $\mu$ be the vertical distance between the middle interpolation point $\left(x_{2}, u_{2}\right)$ and a straight line between the end points $\left(x_{0}, u_{0}\right)$ and $\left(x_{4}, u_{4}\right)$, see Fig. 4. The value of $\mu$ is positive if the interpolation points are above the straight line and negative otherwise. Let $v_{1}$ be the vertical distance between $\left(x_{1}, u_{1}\right)$ and a straight line between $\left(x_{0}, u_{0}\right)$ and $\left(x_{2}, u_{2}\right)$ while $v_{2}$ be the vertical distance between $\left(x_{3}, u_{3}\right)$ and a straight line between $\left(x_{2}, u_{2}\right)$ and $\left(x_{4}, u_{4}\right)$. Both $v_{1}$ and $v_{2}$ are positive if their respective interpolation points are above their respective straight lines and negative otherwise. Then the stretching parameters $d_{1}$ and $d_{2}$ are $\nu_{1} / \mu$ and $\nu_{2} / \mu$, respectively. An illustration of this calculation is presented in Fig. 4.

To see how the local values of $d$ vary in space (or time), we applied this algorithm on a 1D intersections of DNS velocity field of STBL (see Section 3.1 for details of this simulation) [33]. Figure 5a shows the local values of $d$. We see a significant variation of $d$, with some values outside the interval $(-1,1)$, due to the intermittent nature of turbulent velocity fields. To verify the applied procedure, we reduced the resolution of the signal from $\Delta$ to $2 \Delta$ and apply FIT (as described in Section 2.1) once with local values of $d$ as given in Fig. 5a. As expected, the FIT constructed signal is identical with the original one, see Fig. 5 b.

As described in Section 2.1, to assure continuity of the reconstructed signal in the limit of $n \rightarrow \infty$ reconstruction steps, $d$ should take values within the interval $(-1,1)$. In order to satisfy this condition, we further place a constraint $|d| \leq 1$ in the calculation of local stretching parameters and neglect $d$ values outside this interval.

\section{Test Cases}

The stratocumulus clouds have been a major focus of research in the atmospheric turbulence community because of its huge impact on the Earth's radiative balance. This type of clouds covers about one-quarter of the Earth's surface. The turbulence is driven by convective instabilities and large shear forcings [38, 49, 50]. Below, we provide a brief description of DNS, POST airborne campaign and LES of stratocumulus cloud top.

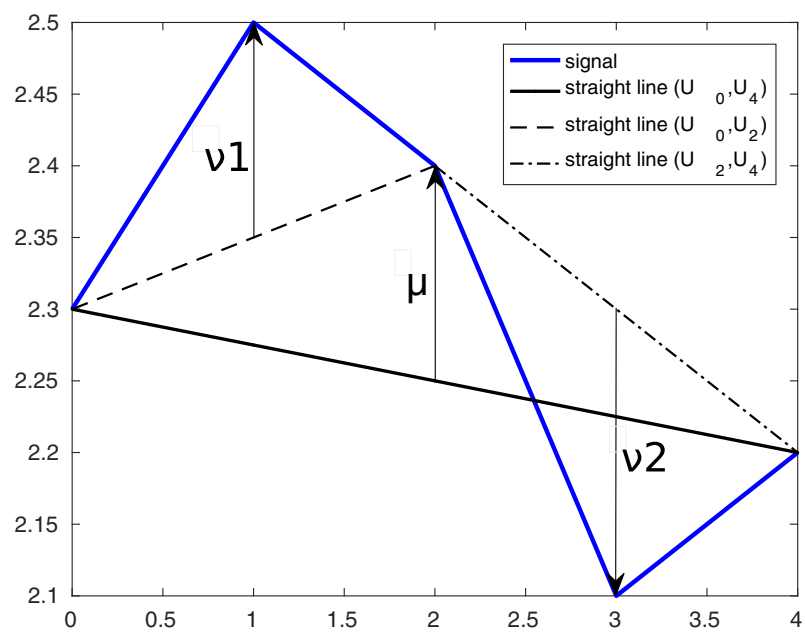

Fig. 4 Illustration of the stretching parameter calculation 


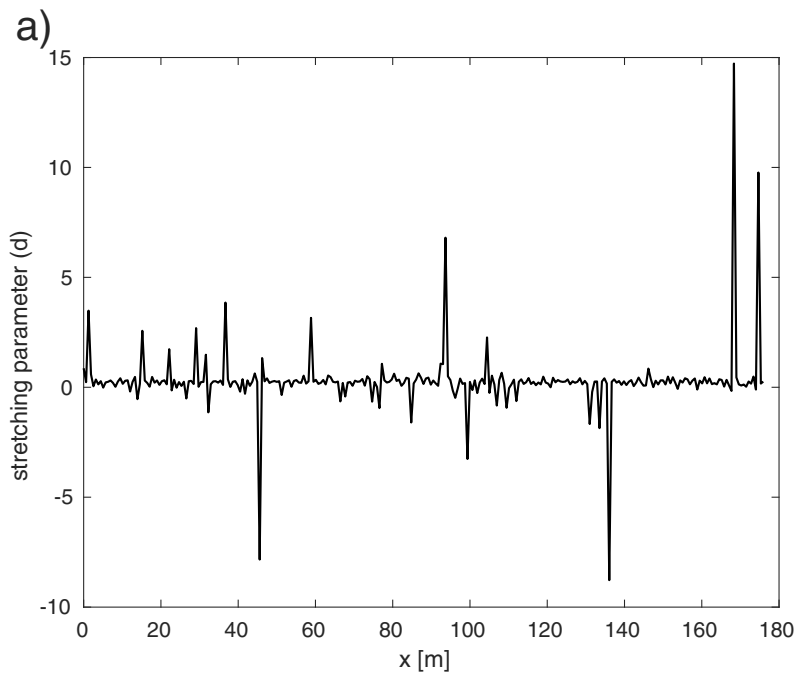

b)

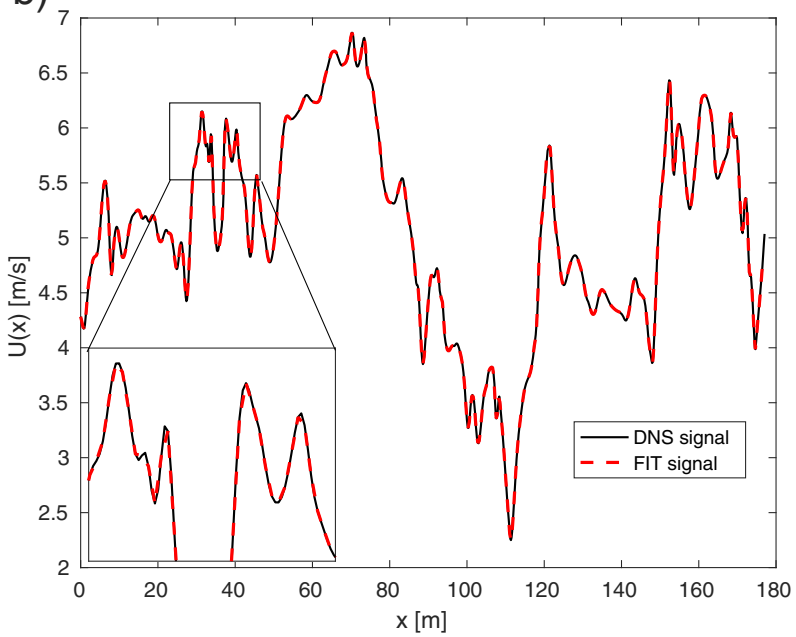

Fig. 5 a Variability of local stretching parameters $d$ in 1D DNS velocity signals b 1D DNS velocity signals showing the original and the FIT reconstructed signal using local values of $d$ in a

\subsection{DNS of stratocumulus-top boundary layer}

The DNS aim to mimic the top of the stratocumulus cloud (see Fig. 6). This restricted region was considered uniquely so as to avoid the huge computational cost required to simulate the whole boundary layer. The simulation settings aim to reproduce the cloud dynamics, as measured during the second Dynamics and Chemistry of Marine Stratocumulus (DYCOMSII) field study [51], with $R e$ number being the only parameter which does not match the experimental data, due to extensive numerical cost of the DNS. Details of the simulations can be found in [50].

The cloud-top mixing layer has two infinite horizontal layers of moist air: the lower region, which is cool and saturated and the upper region, which is warm and unsaturated. 

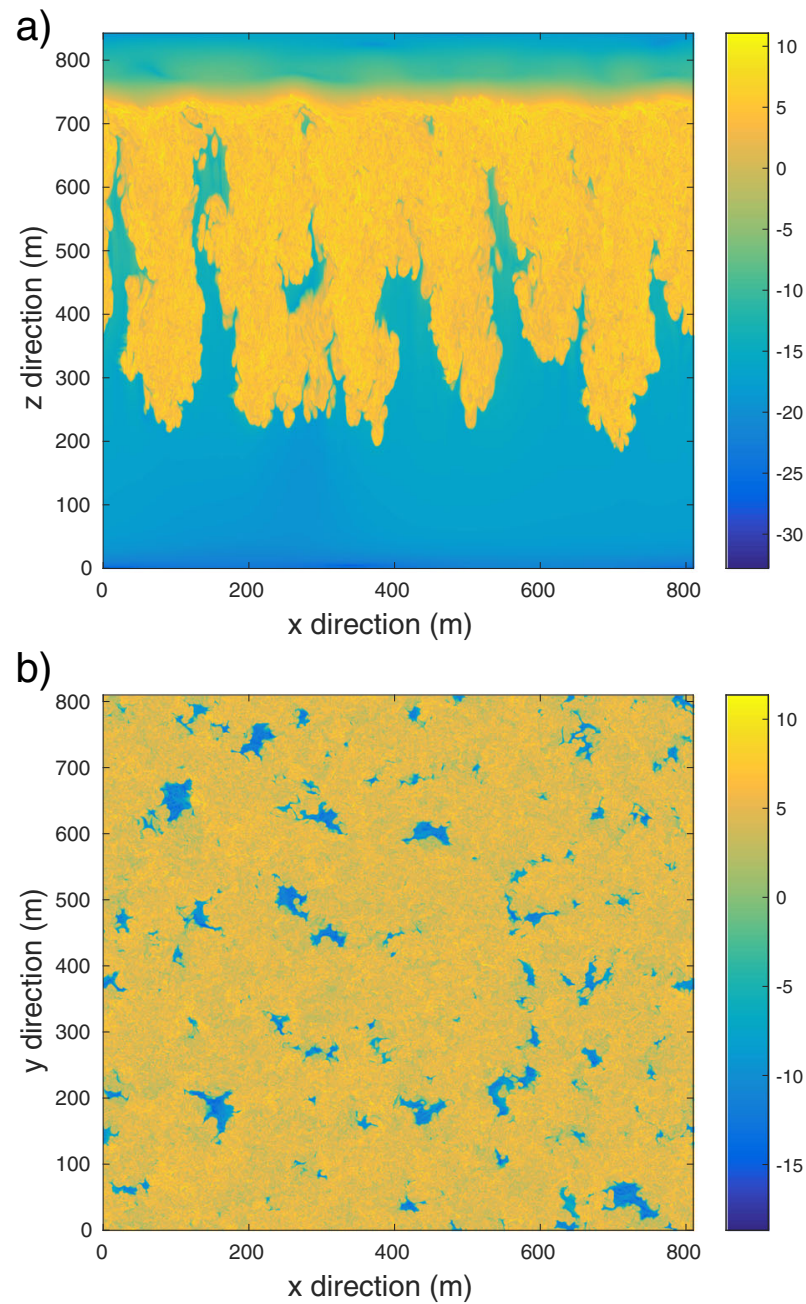

Fig. 6 Logarithm of enstrophy $\left(\Omega=1 / 2|\omega|^{2}\right.$ where $\omega$ is the vorticity field). a Vertical cross section at $y=405 \mathrm{~m}$. b Horizontal cross section at $z=550 \mathrm{~m}$ [50]

Cloud turbulence is driven by convective instability caused by longwave radiative cooling of the upper region of the cloud. Other sources of forcings are wind-shear effects induced by a velocity variation of $3 \mathrm{~m} \mathrm{~s}^{-1}$ along the cloud-top region and the evaporative cooling induced by the mixing of cloudy and environmental air [52]. The upward radiative flux $F_{0}$ which characterizes radiative cooling and the radiative extinction length $L_{0}$ are estimated from the DYCOMS-II field study as, respectively $F_{0}=70 \mathrm{~W} / \mathrm{m}^{2}$ and $L_{0}=15 \mathrm{~m}$. With this, the reference buoyancy flux equals $B_{0}=F_{0} g /\left(\rho c_{p} T_{0}\right)=0.002 \mathrm{~m}^{2} / \mathrm{s}^{3}$, where $g$ is the gravitational acceleration, $\rho$ is the density of the air, $c_{p}$ denotes the air's heat capacity and $T_{0}$ is the reference temperature. The Reynolds number based on these scales is $R e=$ $U_{0} L_{0} / v=800$ where the reference velocity scale $U_{0}=\left(B_{0} L_{0}\right)^{1 / 3}=0.3 \mathrm{~m} \mathrm{~s}^{-1}$. Here the kinematic viscosity $v=0.0056 \mathrm{~m}^{2} / \mathrm{s}$ is almost 400 times larger than the air's viscosity. For a later comparison with LES study and measurements, we calculate Reynolds number based 
on the Taylor microscale $\operatorname{Re}_{\lambda}=u^{\prime} \lambda / v$, where $u^{\prime}$ is the turbulence intensity. The Taylor microscale $\lambda$ is related to the dissipation of the turbulence kinetic energy $\varepsilon$ by the formula $\lambda=\left[15 v u^{\prime 2} / \varepsilon\right]^{0.5}$, see [53]. In the in-cloud region $u^{\prime} \approx 0.3 \mathrm{~m} / \mathrm{s}$ and $\varepsilon \approx 0.6 \cdot 10^{-3} \mathrm{~m}^{2} / \mathrm{s}^{3}$, see [50], which gives $\lambda \approx 3.5 m$ and $R e_{\lambda} \approx 190$.

The horizontal size of the computational domain is $54 L_{0}$ which is discretized with $5120 \times 5120 \times 2048$ points in $x$ - (streamwise), $y$ - (spanwise) and $z$ - (vertical) directions. This ensures the fine resolution of the flow down to the smallest dissipative eddies with a characteristic size $\eta_{0} \simeq 10 \mathrm{~cm}$. The simulation is statistically homogeneous in the horizontal $x$ and $y$ directions. The statistics at these horizontal planes depend on the vertical coordinate $z$ and time $t$. Figure 6 shows the vertical cross-section (at $y=405 \mathrm{~m}$ ) and horizontal cross-section (at $z=550 \mathrm{~m}$ ) of the logarithm of enstrophy (i.e. $\Omega=1 / 2|\omega|^{2}$ where $\omega$ is the vorticity field). Turbulence statistics, that is, the mean streamwise velocity component, r.m.s. of horizontal $\left(u^{\prime}, v^{\prime}\right)$ and vertical $\left(w^{\prime}\right)$ velocity fluctuations and the budget of turbulence kinetic energy are presented in Fig. 7.

\subsection{POST airborne data}

To investigate further the variability of the stretching parameter in high Reynolds turbulence, we compute $d$ from the airborne data. We use high-resolution in situ measurements of wind velocity fluctuations (time signals) from one of the flight segments in the stratocumulus top boundary layer. This segment was a part of flight 13 of the POST research campaign [37, 39] carried out in the vicinity of Monterey Bay in July and August 2008 (the data are available online via https://www.eol.ucar.edu/projects/post/). The signal's sampling frequency was $40 \mathrm{~Hz}$ (corresponding to $\sim 1.4 \mathrm{~m}$ spatial resolution) and the duration was $T=438.75 \mathrm{~s}$. The magnitude of the vector difference between the wind and aircraft velocity, averaged over the track vector, was $55 \mathrm{~ms}^{-1}$ and the turbulence intensity $u^{\prime}=0.28 \mathrm{~ms}^{-1}$. Turbulence kinetic energy dissipation rate was estimated from the power spectral density and the second-order structure function in Ref. [38]. For the flight 13 the average value in the well-mixed cloud top layer was $\varepsilon=0.55 \cdot 10^{-3} \mathrm{~m}^{2} / \mathrm{s}^{3}$. The kinematic viscosity at the flight height was $v=$ $1.46 \cdot 10^{-5} \mathrm{~m}^{2} / \mathrm{s}$. With this, the Taylor microscale equals, approximately $\lambda \approx 0.19 \mathrm{~m}$ and $R e_{\lambda} \approx 3900$ is around 20 times larger than in the DNS described in Section 3.1.

\subsection{LES of stratocumulus-top boundary layer}

The large eddy simulation of stratocumulus top boundary layer for POST flight 13 [36] was performed with a simplified version of 3-D non-hydrostatic anelastic Eulerian-semiLagrangian (EULAG) model [54] without sub-grid scale (SGS) model (also called implicit LES). In implicit LES, the truncation terms of the numerical scheme account for the effect of unresolved turbulence. In previous works [55-57], it was shown that implicit LES performs comparable to conventional LES (with SGS model). The code solves the three velocity components $(\vec{u}=(u, v, w))$ in $x-, y-$ and $z$ - directions with other atmospheric variables such as potential temperature $\theta$ etc. The velocity components were advected using the Multidimensional Positive Definite Advection Transport Algorithm (MPDATA) [58].

The computational domain is $3 \mathrm{~km}$ in $x$ and $y$ directions and $1.2 \mathrm{~km}$ in $z$ direction with a grid resolution of $5 \mathrm{~m}$ in each directions. The flow is periodic across the horizontal boundaries and impermeable free slip condition is applied at the upper boundary. The lower boundary is impermeable with partial slip conditions imposed through near-surface momentum fluxes. The initial vertical profile of $u$ and $v$ velocity components were set to the geostrophic wind $U_{g}=5.0 \mathrm{~m} \mathrm{~s}^{-1}$ and $V_{g}=-7.0 \mathrm{~m} \mathrm{~s}^{-1}$, respectively. The time step $\delta t$ 
Fig. 7 Some statistics of velocity field in the cloud-top mixing layer. The upper horizontal line indicates the height of minimum buoyancy flux (horizontal plane $z=585 \mathrm{~m}$ ) while the lower horizontal black line indicates the height of maximum buoyancy flux (horizontal plane $z=540 \mathrm{~m}$ ) [50]

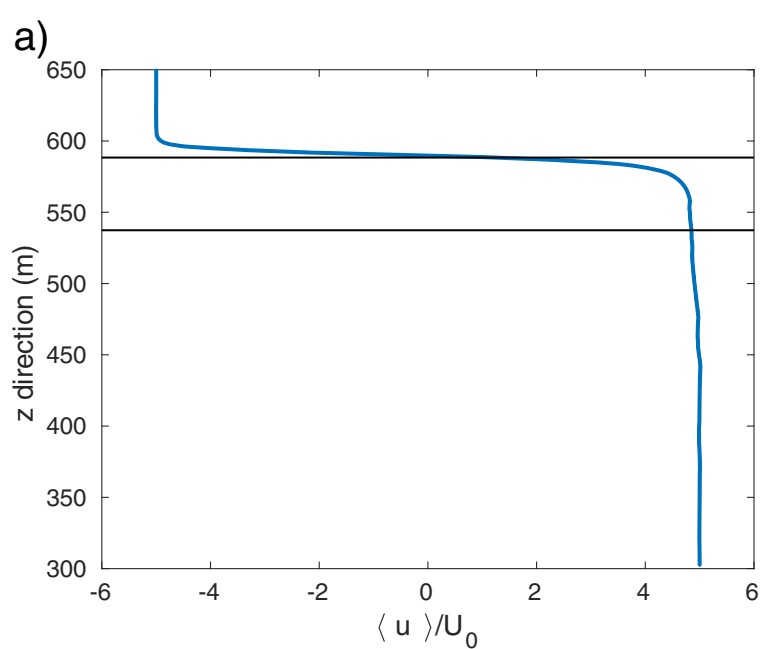

b)

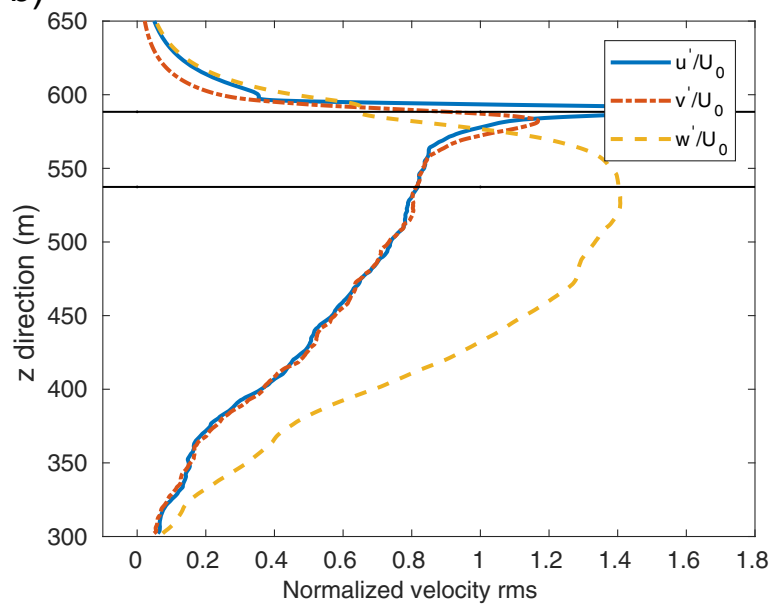

c)

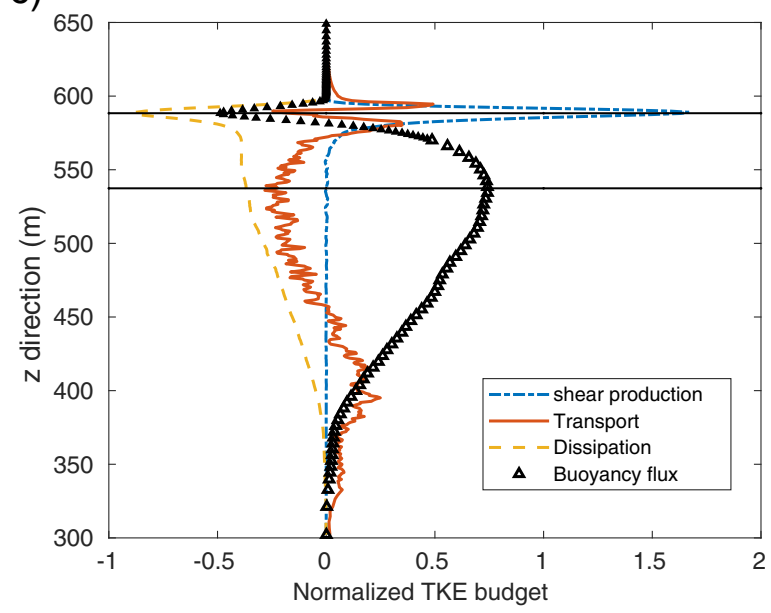


was chosen such that the maximum value of the Courant number throughout the simulation period and computational domain is $\sim 0.5$. For details, readers are referred to Pedersen et al. [36]. Figure 8 shows $u$ velocity component along the horizontal and vertical cross-sections of the flow field. The cloud top region is placed at the vertical height $z \sim 700 \mathrm{~m}$. Above this region is the free troposphere, which is warm and unsaturated with low turbulence intensity while the region below the cloud top is moist, saturated with high turbulence intensity. Vertical profile of mean velocity field and r.m.s. of horizontal and vertical velocity fluctuations are shown in Fig. 9.

\section{Probability Distribution Function of the Stretching Parameter}

In this section, we analyse available data of velocity field from numerical simulations and experiment described in Section 3. We extract stretching parameters, as discussed in
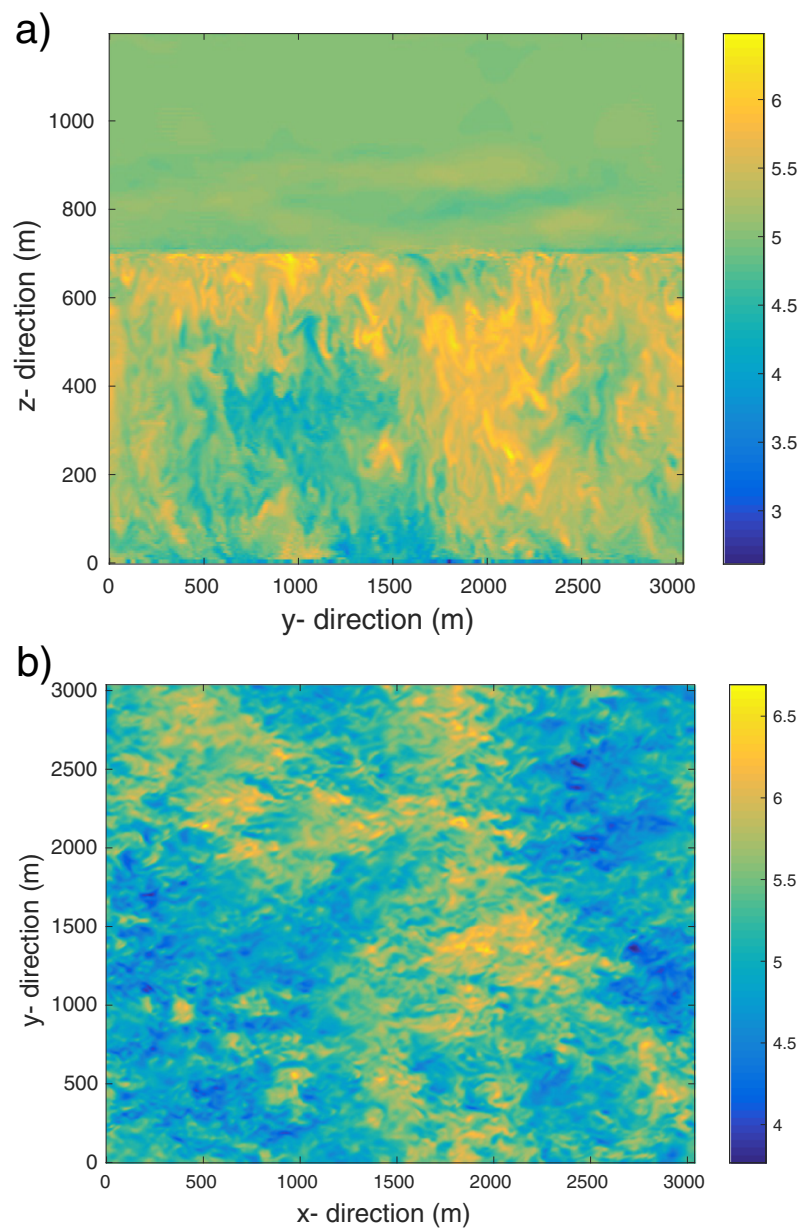

Fig. 8 Velocity component $u$ of LES field. a Vertical cross section at $y=1595 \mathrm{~m}$. b Horizontal cross section at $z=595 \mathrm{~m}$ 
a)

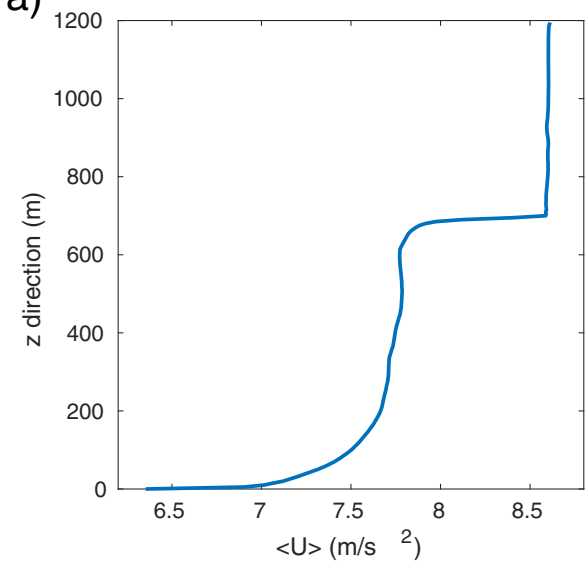

b)

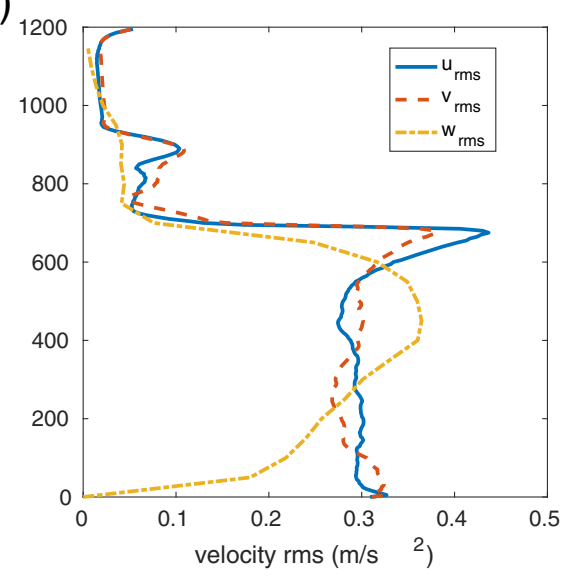

Fig. 9 Statistics of LES velocity field $\mathbf{a}$ vertical profile of mean velocity $\mathbf{b}$ vertical profile of velocity rootmean-square

Section 2.2 and calculate the PDFs of the module of $|d|$, denoted as $f(|\mathrm{~d}|)$. Here, $\mathrm{d}$ stands for the sample space variable of the stretching parameter $d$.

\subsection{DNS of stratocumulus-top mixing layer}

For the DNS data described in Section 3.1, we use horizontal profiles of the $u, v$ and $w$ components of velocity at a height corresponding to the in-cloud region $(z=550 \mathrm{~m})$. First, we investigate the variability of $d$ as 1-D intersections of DNS velocity field are filtered successively to wavenumber in the inertial range. Starting with the fully resolved DNS field with the grid spacing equal to $\eta_{0}$, we reduce the resolution to $2 \eta_{0}$ by using a low-pass filter and calculate the local values of $d$ for the filtered velocity field. Then, the velocity signal is filtered to a grid resolution of $4 \eta_{0}$ etc., the cut-off wavenumber is decreased, until the resolution matches the inertial-range (at about $16 \eta_{0}$ to $128 \eta_{0}$ ). After each filtering, the local values of $d$ are extracted. The low-pass filter used is the finite impulse response (FIR) filter of order 30 designed using the Hamming window method [59]. This is done with decimate function in MATLAB ${ }^{\circledR}$ software. We use the decimation low-pass filter because it is constructed to downsample the signal (i.e reduce the number of grid points) and guard against aliasing. The downsampling feature of the filter was important to have a reconstructed signal with the same number of grid points as the original (DNS, LES or POST airborne) signal. We calculate the local estimate of $d$ with the algorithm explained in Section 2.2. Stretching parameter values outside the interval $(-1,1)$ were neglected and absolute value of $d$ was used to calculate its PDF. Since the flow is statistically homogeneous over horizontal planes similar results were obtained for 1D intersections of velocity field calculated either in $x$ - or $y$ - directions.

Figure 10 presents the PDF of $|d|$ and average fractal dimension of DNS velocity signals at different grid resolutions. In Fig. 10a, the PDFs change significantly for the first four successive filtering steps but seem to be self-similar when filtered successively to inertialrange wavenumbers (at steps 4 to 7). All three velocity components give similar profiles of the PDF of the stretching parameter. The average fractal dimension, calculated according to Eq. 8, decreases to 5/3 inertial range scaling, as seen in Fig. 10b. The calculated vertical 

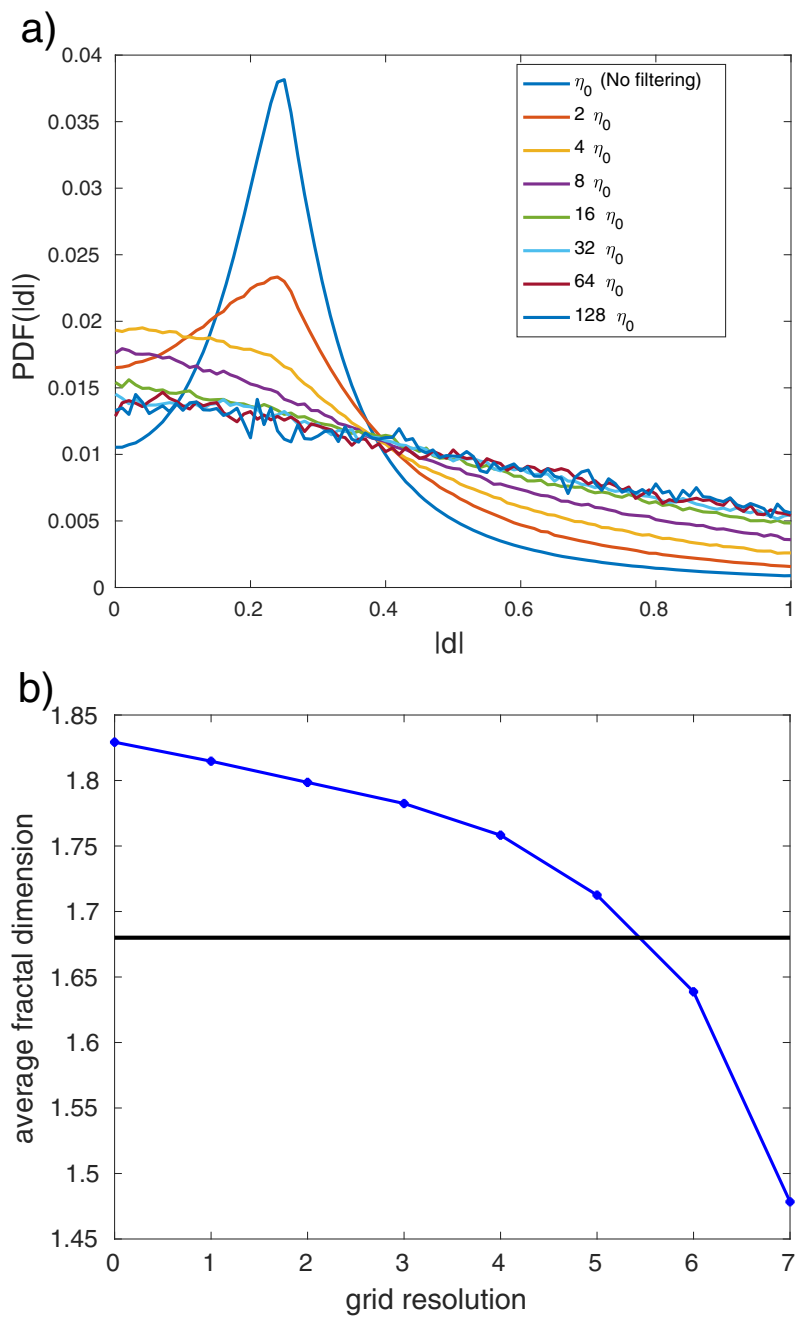

Fig. 10 a PDFs of the absolute value of stretching parameter for horizontal profile of DNS velocity at different resolutions. b The average fractal dimension for horizontal profile of DNS velocity at different resolutions. The black line shows the value corresponding to the $-5 / 3$ inertial-range scaling

profile of averaged $|d|$ across the STBL (see Fig. 11b) is smaller than 0.6 or 0.7 reported in Ref. [31] even in the core cloud region (at $z \approx 550 \mathrm{~m}$ ). We expect this result follows from the presence of external intermittency (laminar regions will give zero or close to zero local values of $d$ ). Even in the in-cloud region the volume fraction occupied by turbulent flow is smaller than one and equals approximately $\gamma=0.9-0.95$, where $\gamma$ is the intermittency parameter, see [60]. Due to small values of $\gamma$ in the outer-cloud regions, the average value of $d$ also decreases therein to, approximately 0.25 , see Fig. $11 \mathrm{~b}$.

In Ref. [20], it was shown that a fractal signal will only dissipate energy in the limit of small viscosity if $|d|>0.5$. So as to retain dissipative properties, we neglect $|d|<0.5$ in further sub-grid scale reconstruction in Section 5.1. If only $|d|>0.5$ is considered, the average stretching parameter will be similar to values obtained in Ref. [31]. 


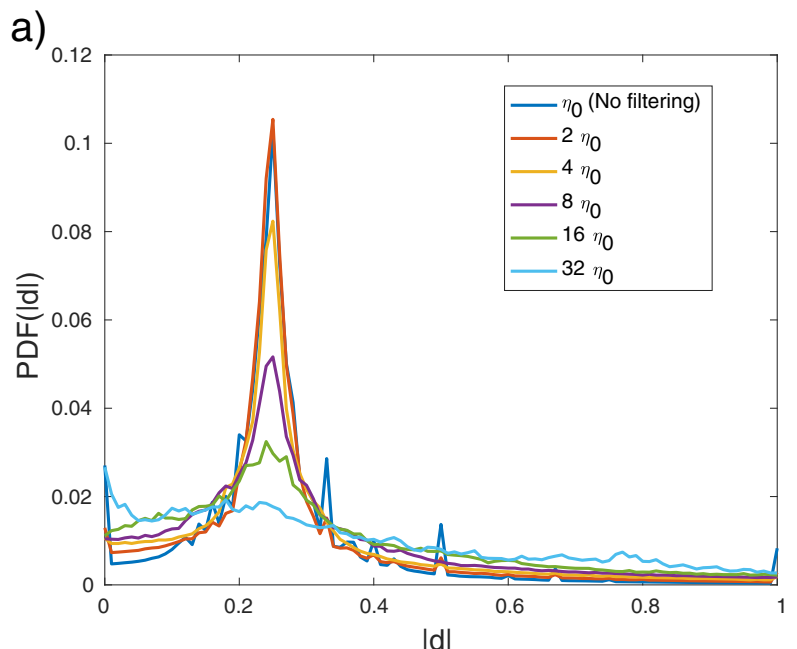

b)

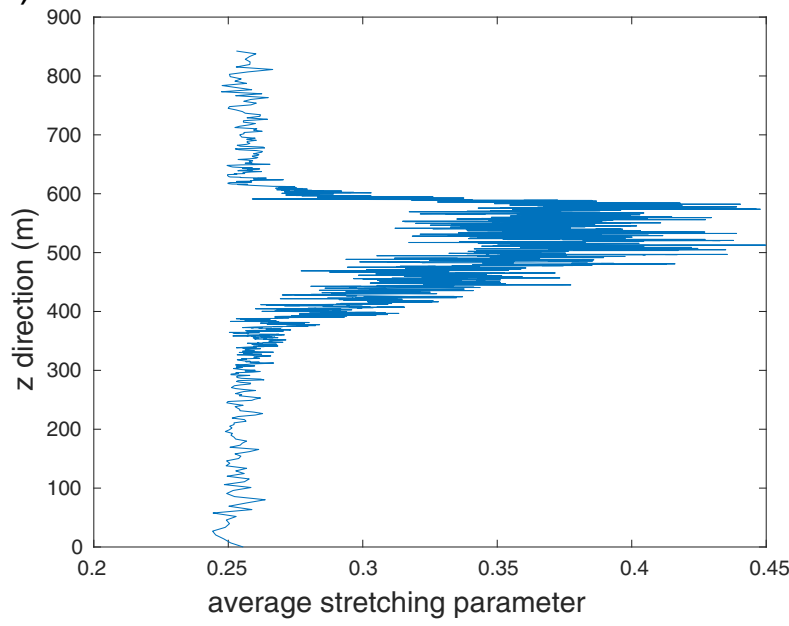

Fig. 11 a PDFs of the absolute value of stretching parameter for vertical profile of DNS velocity at different resolutions. Inertial-range wavenumbers correspond to iteration 4 and $5 \mathbf{b}$ The average stretching parameter for vertical profile of DNS velocity

No self-similarity of PDFs of $|d|$ is observed in the vertical $z$ - direction, see Fig. 11a. Here, velocity field was filtered to a grid resolution of $16 \eta_{0}$ or $32 \eta_{0}$. This is due to the anisotropy of turbulence caused by large scale shear production and buoyancy. Similar conclusion was made by Marchioli et al. [2, 3] where the locally computed stetching parameters varied significantly in the wall-normal direction. Also, in that study, the average stretching parameter was lower than that obtained experimentally from homogeneous isotropic turbulence.

We observe no significant variation in the horizontally calculated average of $d$ in the in-cloud region - at $450 m \leq z \leq 550 m$ (see Fig. 11b). Hence, the remaining analysis is based on the horizontal profiles in this region, where turbulence is close to isotropic. 
We also observe that probabilities of having positive or negative stretching parameter are equal.

\subsection{Comparison with LES and POST data}

To investigate the variability of the stretching parameter in high Reynolds turbulence, we compute $d$ from LES field and from the POST airborne dataset using the Mazel and Hayes' algorithm described in Section 2.2. We compare PDF of $|d|$ from LES and POST data with the respective profile from the DNS. DNS velocity field was filtered with the decimate function [59] (described in Section 4.1) to a spatial resolution of $16 \eta_{0}$ (equivalent to $1.6 \mathrm{~m}$ ). For LES, we use the horizontal profile of $u$ velocity component at $z=595 \mathrm{~m}$ (corresponding to the in-cloud region with the maximum turbulent intensity) to estimate stretching parameters. Numerical scheme of LES introduces a spurious damping of the energy of the smallest resolved scales [24]. In order to remove these effects, we filtered the LES velocity field with the decimate function (described in Section 4.1), to a grid resolution of $20 \mathrm{~m}$ (corresponds to inertial-range wavenumber) from its $5 \mathrm{~m}$ grid resolution. Another possibility, instead of filtering, would be to recover the energy of the smallest scales using the approximate deconvolution method - ADM (see [24] for details). Combining the ADM and the FIT is, however, left for further work and we will not address this issue here. We did not filter the POST airborne data since its sampling frequency is within the inertial-range. All the three $u, v$ and $w$ components of POST velocity dataset were combined to form a large dataset since their individual PDF of $|d|$ were similar.

As seen in Fig. 12 comparison between LES and DNS profiles is very good, in spite of different Reynolds numbers of the two simulations. The PDF of $|d|$ from POST data seems to oscillate around the respective DNS profile. Some differences observed may be due to the effect of large scale tendencies on measured airborne data and small scale measurement noise. Based on the results shown in Fig. 12, we can conclude the PDF of the stretching parameter in the inertial range is a universal function, independent of the Reynolds number.

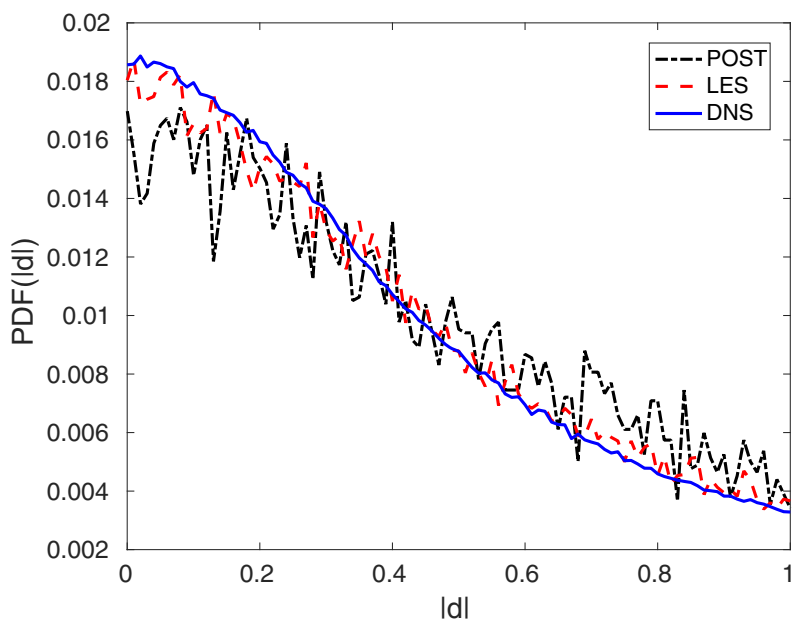

Fig. 12 Probability distribution of the absolute value of the stretching parameter $|d|$ from filtered DNS, LES velocity signals of stratocumulus cloud-top and POST data. DNS and LES velocity fields were filtered with wavenumber within the inertial range 


\section{Results}

\subsection{Fractal interpolation of filtered DNS}

First, we filter $1 \mathrm{D}$ horizontal intersections of DNS velocity field at $z=550 \mathrm{~m}$ to a spatial resolution of $16 \eta_{0} \approx 1.6 \mathrm{~m}$ (which is within the inertial range) using the decimation function described in Section 4.1. We next reconstruct sub-filter scales back to the resolution $\eta_{0}$ using FIT. For this, we select the stretching parameter from its PDF calculated from the DNS data, see Fig. 12, using the inverse transform sampling method [61]. The procedure is briefly indicated as following

1. Calculate cumulative distribution function $F(|\mathrm{~d}|)$ from the $\operatorname{PDF} f(\mid \mathrm{d}])$, as

$$
F(|\mathrm{~d}|)=\int_{0}^{|d|} f(s) d s .
$$

2. Calculate the inverse function $F^{-1}(\mathrm{y})$.

3. If $y$ is a random number from uniform distribution $[0,1]$ then $d=F^{-1}(y)$ is a random number from the investigated PDF.

Apart from the mathematical constraint $|d| \leq 1$, which assures the continuity of the reconstructed signal at $n \rightarrow \infty$ reconstruction steps [45], the second constraint, discussed in Section 3.1 was related to the dissipative properties of the signal [48] and reads $|d|>$ 0.5 . Hence, in practice, in the reconstruction process, we only retained the values $|d|$ that were larger than 0.5 . If selected $|d| \leq 0.5$, the procedure was repeated and another random value was chosen, till the condition $0.5<|d| \leq 1$ was satisfied. Next, the sign of $d$ was selected randomly, such that the positive and negative $d$ have equal probabilities. Under such assumptions, the ensemble average $\langle|d|\rangle$ is comparable to values reported in Ref. [31] and the scaling of the reconstructed energy spectra is close to the theoretical $k^{-5 / 3}$. We checked that using only one constraint, $|d| \leq 1$, led to underprediction of the reconstructed spectra. Hence, the choice $0.5<|d| \leq 1$ seems the most favourable as it is supported by the theoretical constraints $[19,48]$ and gives satisfactory results.

The inertial-range scale invariance is the property that directly relates to the idea of fractality of velocity field. As seen in Fig. 10a, profiles of PDF $f(|\mathrm{~d}|)$ collapse into one curve only for cut-off wavenumbers from the inertial range. For this reason, the fractal reconstruction of the dissipative part of the spectrum is not justified, as no self-similarity is observed there. Instead, in the FIT reconstruction process, the inertial range was extended down to $\eta_{0}$ and properties of such an artificial velocity field were investigated. The lack of the dissipative range could be a possible drawback if Lagrangian particles are tracked in the reconstructed field. This would concern especially the motion of small-inertia particles which are correlated with smaller eddies [14]. However, in practice, FIT in numerical simulations of high- $R e$ flows will be restricted to a few reconstruction steps, due to increased computational time. With this, reconstruction of the signal down to $\eta_{0}$ will not be possible and the smallest resolved scales will belong to the inertial range.

Figure 13 shows the longitudinal energy spectra $E_{11}(k)$ (for $u$ velocity component along $x$ direction) of DNS, filtered and FIT-reconstructed velocity field for the three investigated versions of the model: with $d= \pm 2^{1 / 3}$ as originally proposed by Scotti \& Meneveau [20], with $d_{1}=-0.887, d_{2}=-0.676$ as proposed by Basu et al. [30], and with the new proposal with random $d$. As it is observed, in case of constant values of $d$, energy spectra exhibit periodic modulations (see also figures 7 and 8 of [20]). Basu et al. [30] avoid this modulation by applying the discrete Haar wavelet transform. FIT energy spectrum with constant values 


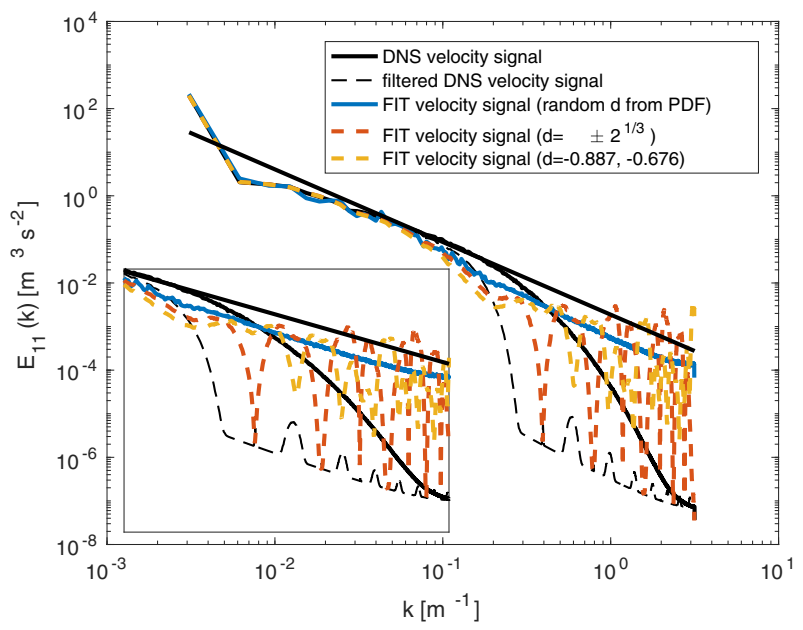

Fig. 13 Longitudinal energy spectra of $u$ velocity component for DNS and FIT with constant stretching parameter $d= \pm 2^{-1 / 3}$, with constant stretching parameters $d=-0.887$ and $d=-0.676$ and with random stretching parameters from calculated PDF

of $d= \pm 2^{1 / 3}$ is much lower in some wave-numbers than the $-5 / 3$ inertial-range scaling (especially at wavenumbers close to the cut-off scale), although the upper envelope qualitatively follows the $-5 / 3$ inertial-range scaling. The FIT energy spectrum reconstructed with constant values of $d=-0.887,-0.676$ has similar properties as the Scotti \& Meneveau approach, except that the range between the upper and lower envelope of the spectrum is somewhat smaller. The FIT energy spectrum reconstructed with random values of $d$ follows the inertial-range scaling closer and shows no periodic modulation.

Next, we investigated statistics of velocity increments at two different points $\mathbf{u}(\mathbf{x}+\mathbf{r}, t)-$ $\mathbf{u}(\mathbf{x}, t)$. The sample space of velocity increment will be denoted by $\delta \mathbf{u}$ and the distance between points by $r=|\mathbf{r}|$. The tails of the PDFs of velocity increments in the isotropic turbulence $f(\delta u, r, t)$, can be approximated by the stretched exponentials, where the stretching exponent varies monotonically from 0.5 for $r$ in the dissipation range to 2 (which is a Gaussian distribution) for $r$ in the integral scale range, [62]. The non-Gaussianity of the PDFs for small $r$ indicates the presence of the internal intermittency, that is, the probability of extreme events (large velocity differences) at these scales is much higher than predicted by a Gaussian distribution.

Figure 14 presents the PDFs of velocity increment $(\delta u)$ for DNS, filtered DNS and FIT velocity signals with constant and random values of $d$ for $r=128 \eta_{0}$ and $r=64 \eta_{0}$. To increase the size of dataset used to calculate PDFs we considered both longitudinal $(x)$ and transverse $(y) 1 \mathrm{D}$ intersections of the velocity field at $z=550 \mathrm{~m}$ and, additionally, averaged results over all three velocity components.

All three FIT approaches presented in Fig. 14 compare well with the corresponding DNS profiles. PDFs at $r=128 \eta_{0}$ are clearly Gaussian, see [32, 41]. Differences are observed when the distance $r$ decreases to $r=8 \eta_{0}, 4 \eta_{0}$ and $2 \eta_{0}$, see Figs. 15, 16 and 17, respectively. The PDFs of velocity increments are far from Gaussian and slightly skewed. The FIT model with random $d$ provides the best FIT with the DNS at smaller $r$.

We note here that $r=4 \eta_{0}$ and $2 \eta_{0}$ correspond to dissipative-range scales. The intermittency at short $r$ seems to be correctly reproduced, especially by the approach with random 

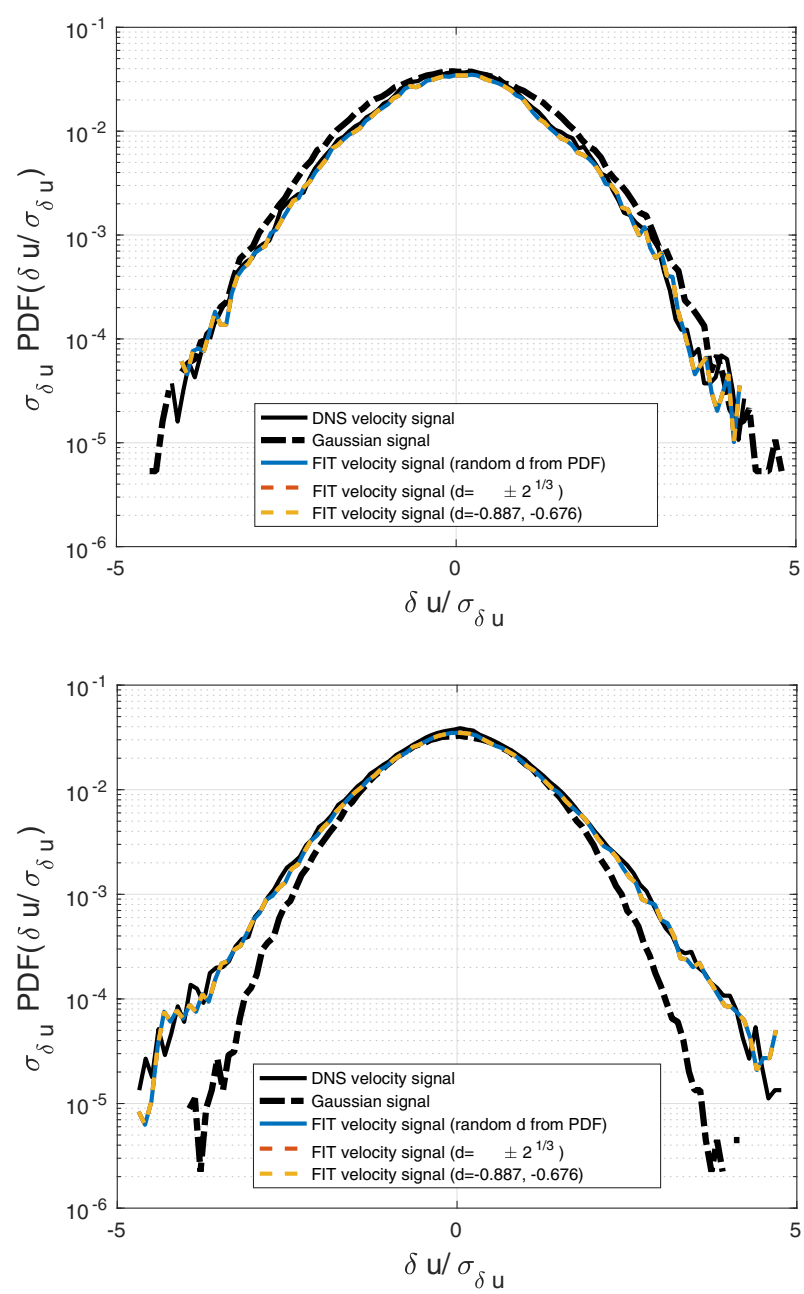

Fig. 14 PDFs of velocity increments of DNS, filtered DNS and FIT fields at $\mathbf{a} r=128 \eta_{0}, \mathbf{b} r=64 \eta_{0}$ showing the Gaussianity of PDFs at large scales

$d$, although in the reconstruction process, the dissipative range is not reproduced in any FIT model. We report here this result which seems interesting, although somewhat unanticipated at first sight.

\subsection{Fractal interpolation of POST airborne data}

In order to test the performance of our sub-grid model on high Reynolds number airborne data, we use $u$ component of velocity field from flight 13 in POST airborne research campaign $[37,39]$. The velocity signal was filtered with the decimation function (described in Section 4.1) from its frequency of $40 \mathrm{~Hz}$ to $10 \mathrm{~Hz}$ (corresponding to the spatial resolution of $5.6 \mathrm{~m}$ ). The filtering was done to eliminate measurements errors at large frequencies. We use this filtered signal as our reference velocity dataset to validate the performance of the 


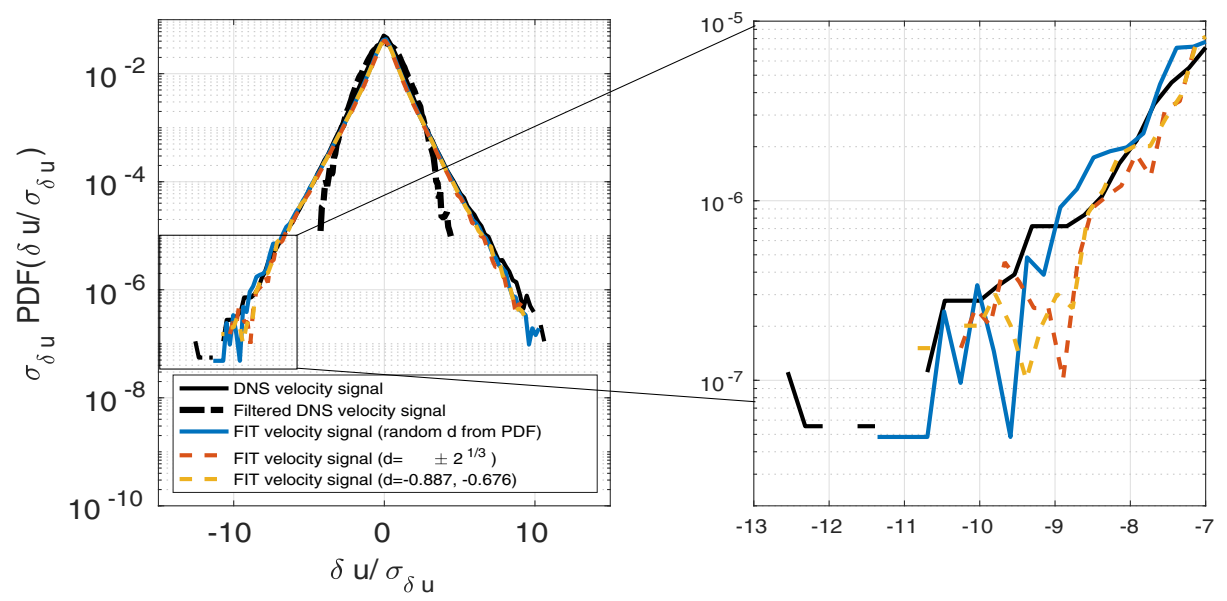

Fig. 15 PDFs of velocity increments of DNS, filtered DNS and FIT fields at $|\mathbf{r}|=8 \eta_{0}$ with constant stretching parameter $d= \pm 2^{-1 / 3}, d=-0.887$ and $d=-0.676$ and random stretching parameters from PDF

FIT model. This dataset has a larger inertial range compared to DNS data analyzed earlier (see Fig. 18a). The reference signal is filtered to a frequency of $2.5 \mathrm{~Hz}$ (about $22.4 \mathrm{~m}$ spatial resolution) and FIT with the random values of $d$ from PDF (calculated from the reference POST signal) is applied to reconstruct the sub-filter part of the dataset. It is important to note that FIT can be carried out in time by replacing the spatial variable $x$ in Eqs. 1 to 6 with time. The frequency spectra for the reference POST, filtered POST and FIT velocity dataset are shown in Fig. 18a. Once again, we observe the approach with random $d$ follows the inertial-range scaling closer than the other two approaches.

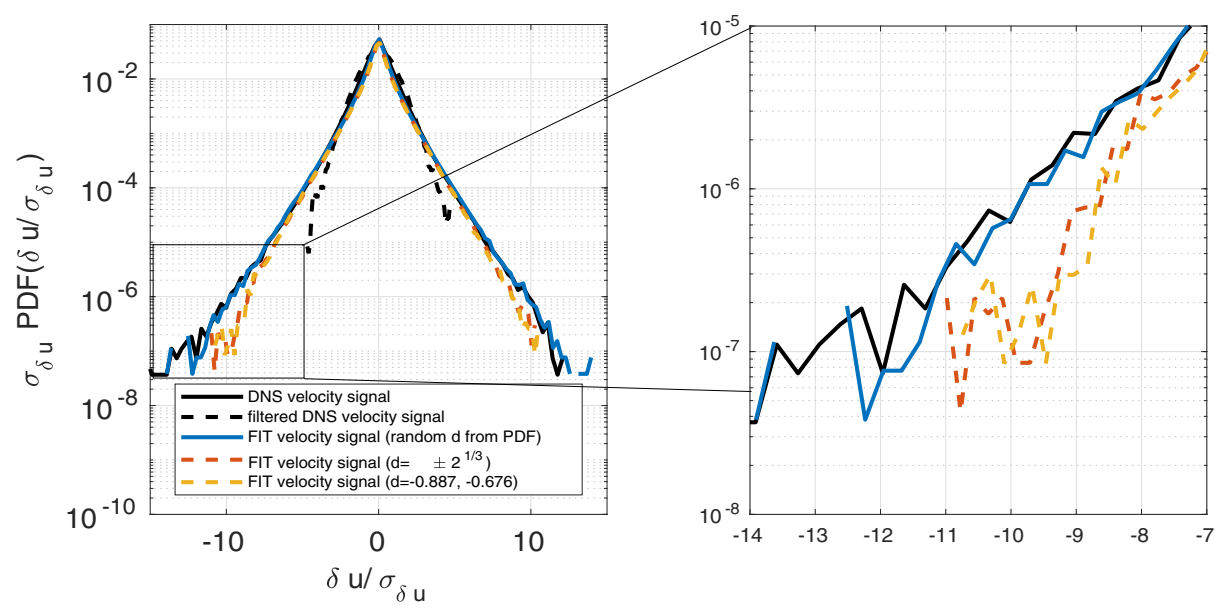

Fig. 16 PDFs of velocity increments of DNS, filtered DNS and FIT fields at $|\mathbf{r}|=4 \eta_{0}$ with constant stretching parameter $d= \pm 2^{-1 / 3}, d=-0.887$ and $d=-0.676$ and random stretching parameters from PDF 


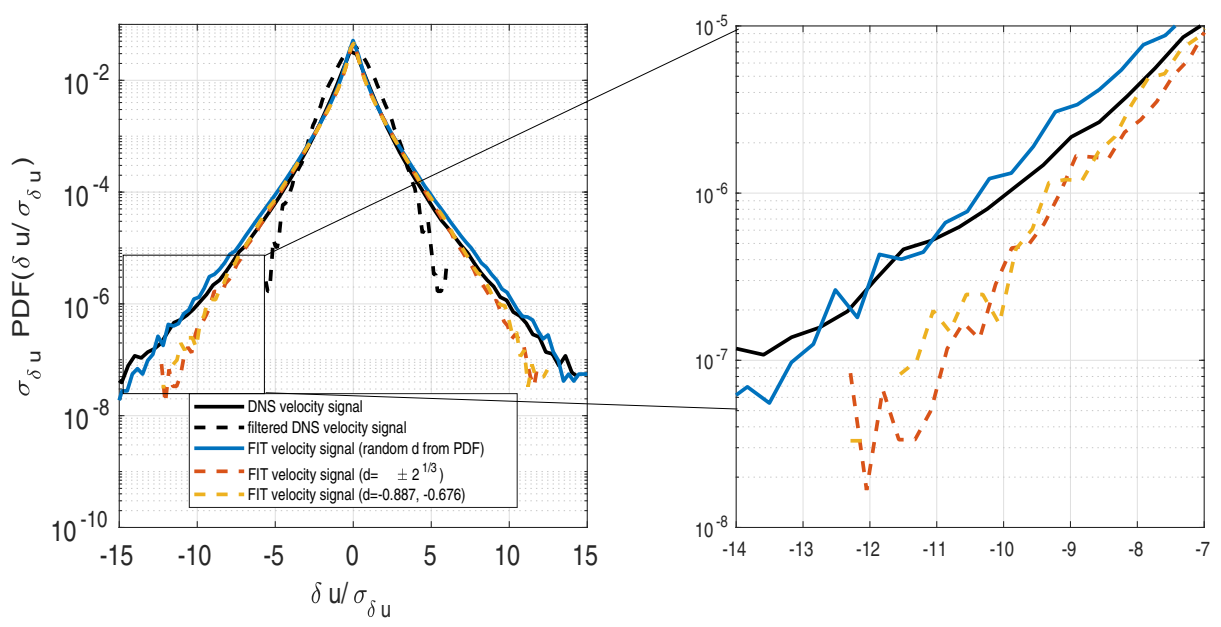

Fig. 17 PDFs of velocity increments of DNS, filtered DNS and FIT fields at $|\mathbf{r}|=2 \eta_{0}$ with constant stretching parameter $d= \pm 2^{-1 / 3}, d=-0.887$ and $d=-0.676$ and random stretching parameters from PDF

Differences in the observed spectra are quantified as follows. Under the local isotropy assumption and within the validity of the Taylor's hypothesis, the energy spectra in the inertial range can be converted to the frequency spectra, see e.g. [63]

$$
S_{t h}=C_{1}\left(\frac{U}{2 \pi}\right)^{2 / 3} \varepsilon^{2 / 3} f^{-5 / 3},
$$

where the constant $C_{1} \approx 0.49, U=55 \mathrm{~ms}^{-1}$ is the true air speed of the aircraft and $\varepsilon$ is the turbulence kinetic energy dissipation rate. The value of $\varepsilon$ can be estimated from the linearleast-squares fit procedure applied in a certian range of frequencies. In the present case, we used $f=0.1 \mathrm{~Hz}-2.0 \mathrm{~Hz}$ and calculated $\varepsilon=4.1306 \times 10^{-4} \mathrm{~m}^{2} / \mathrm{s}^{3}$. Deviations of the spectra reconstructed with FIT from the inertial-range scaling were quantified by $\delta_{S}$ defined as

$$
\delta_{S}=\left[\frac{\int_{f_{c u t}}^{\infty}\left\langle\left(S_{t h}-S(f)\right)^{2}\right\rangle d f}{\int_{f_{\text {cut }}}^{\infty}\left\langle S_{\text {th }}^{2}\right\rangle d f}\right]^{0.5}
$$

where $S(f)$ is the FIT frequency spectrum and $f_{\text {cut }}=2.5 \mathrm{~Hz}$ is the cut-off frequency, i.e. the frequency at which the fractal reconstruction is initiated. We obtained the following values of $\delta_{S}$

$\delta_{S}=0.033$ for FIT procedure with $d= \pm 2^{1 / 3}$

$\delta_{S}=0.049$ for FIT procedure with $d=-0.887,-0.676$

$\delta_{S}=0.026$ for FIT procedure with random $d$

The FIT model with random values of $d$ reproduces the inertial range scaling with the smallest error. Next, we compare the PDF of velocity increments for POST reference data, filtered POST and FIT data with random values of $d$ in Fig. 18b. The velocity increments were calculated as $u(t+\tau)-u(t)$ with $\tau=0.1 \mathrm{~s}$. The PDF of velocity increments for POST reference data and FIT data agree reasonably well. 

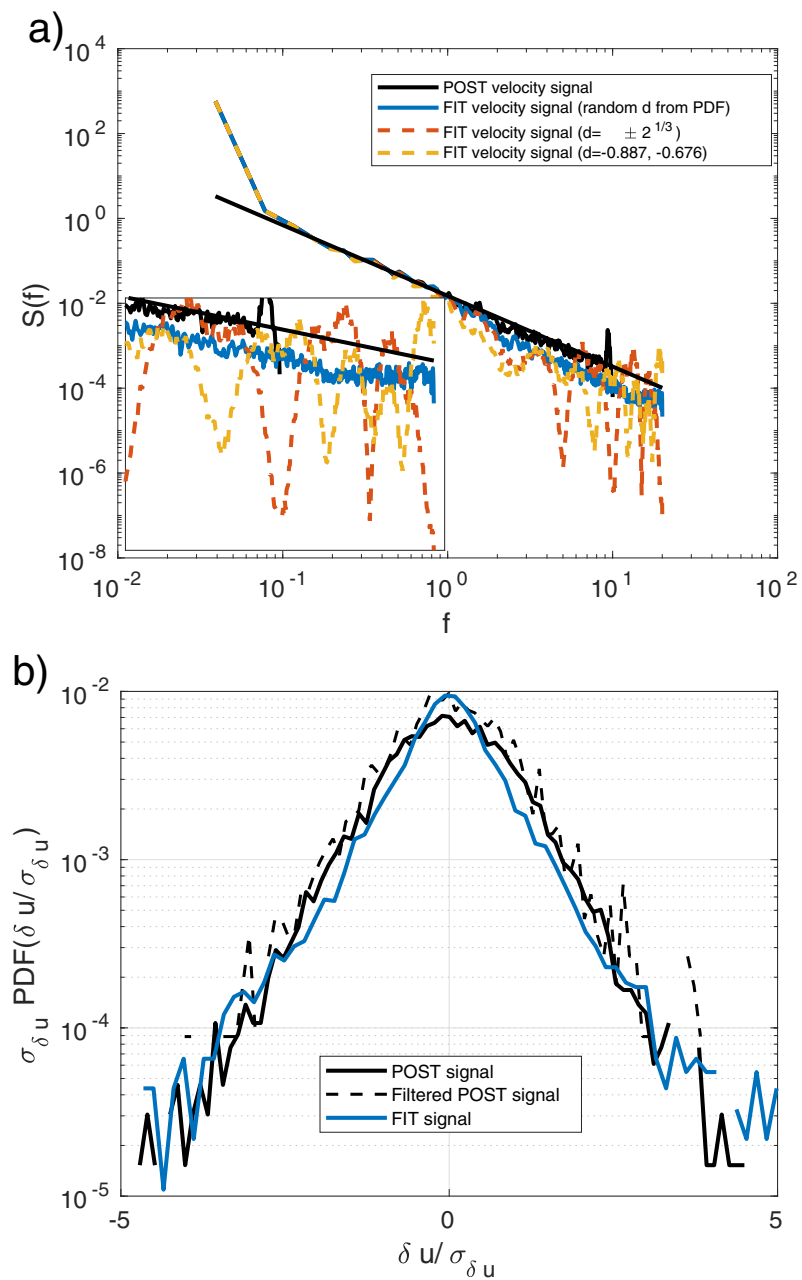

Fig. 18 a Longitudinal energy spectra of POST airborne data and FIT reconstructed signal with constant stretching parameter $d= \pm 2^{-1 / 3}, d=-0.887$ and $d=-0.676$ and random stretching parameters from PDF. b PDFs of velocity increments for POST, filtered POST and FIT signals with random values of $d$

\subsection{3-D fractal interpolation of LES}

Next, fractal interpolation of three components of velocity in 3D using the random values of $d$ (from the PDF shown in Fig. 12) is performed. Each velocity component was filtered with the decimate function (described in Section 4.1) to a grid resolution of $20 \mathrm{~m}$ from its initial $5 \mathrm{~m}$ grid resolution to remove the smallest scales spuriously damped due to numerical diffusion. Then, we perform a 3-D fractal reconstruction of inertial-range scales to a grid resolution of $5 \mathrm{~m}$ (i.e. two reconstruction steps), as described in Section 2.1) without considering any correlations which might exist between the directions.

Figure 19 presents contour plots of the longitudinal $u$ velocity component at $z=$ $595 \mathrm{~m}$ for filtered LES and FIT-reconstructed field. The addition of inertial-range sub-grid structures in FIT velocity is clearly visible. 

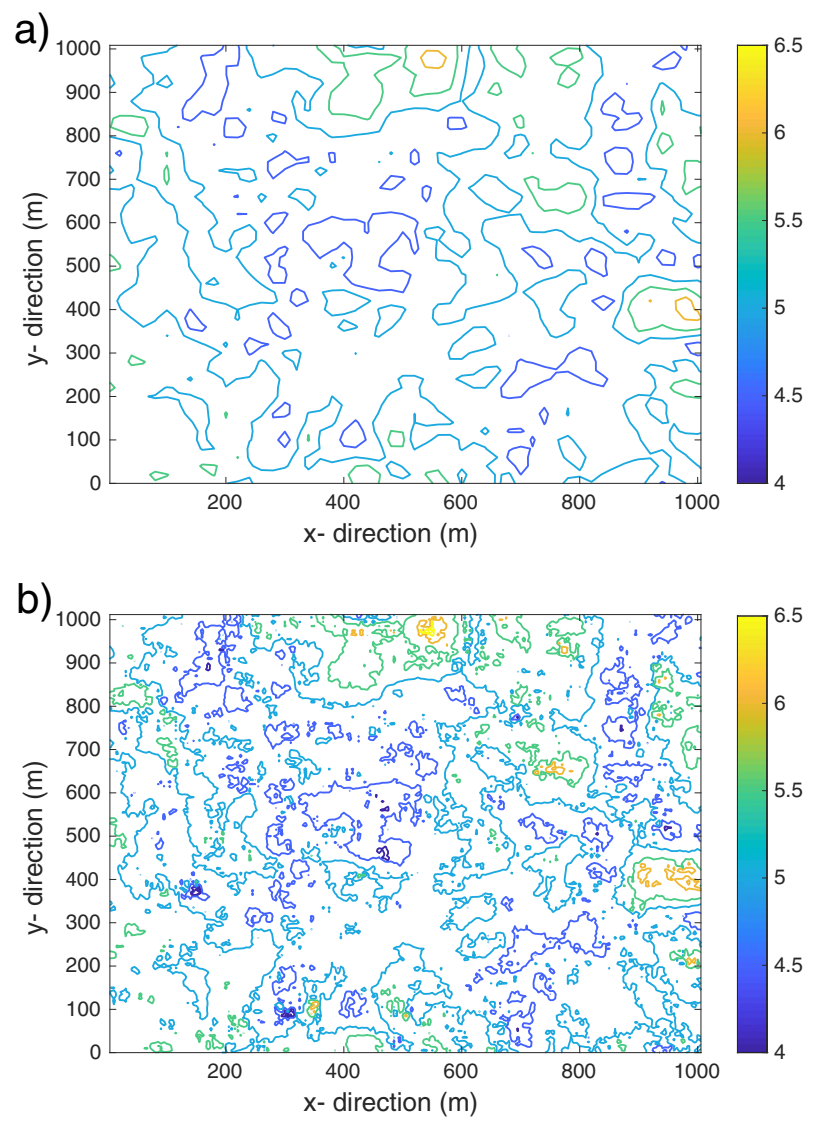

Fig. 19 Contour plot of $u$ velocity field a filtered LES velocity field, $\mathbf{b}$ velocity field of LES with FIT

Energy spectra of this field are presented in Fig. 20 and compared with the two other FIT approaches with constant $d$. The deviations from $-5 / 3$ scaling were calculated from a formula analogous to Eq. 10

$$
\delta_{E}=\left[\frac{\int_{k_{c u t}}^{\infty}\left\langle\left(E_{t h}-E_{11}(k)\right)^{2}\right\rangle d k}{\int_{k_{\text {cut }}}^{\infty}\left\langle E_{t h}^{2}\right\rangle d k}\right]^{0.5},
$$

where $E_{t h}$ is described by formula

$$
E_{t h}=C_{1} \varepsilon^{2 / 3} k^{-5 / 3} \text {. }
$$

With the fitting range $k=0.019 \mathrm{~m}^{-1}-0.044 \mathrm{~m}^{-1}$, the value $\varepsilon=5.0299 \times 10^{-5} \mathrm{~m}^{2} / \mathrm{s}^{3}$ was estimated. The obtained results read

$\delta_{E}=0.40$ for FIT procedure with $d= \pm 2^{1 / 3}$,

$\delta_{E}=0.52$ for FIT procedure with $d=-0.887,-0.676$,

$\delta_{E}=0.28$ for FIT procedure with random $d$.

Here again, FIT with random values of $d$ shows the best agreement with the $-5 / 3$ inertial-range scaling. 


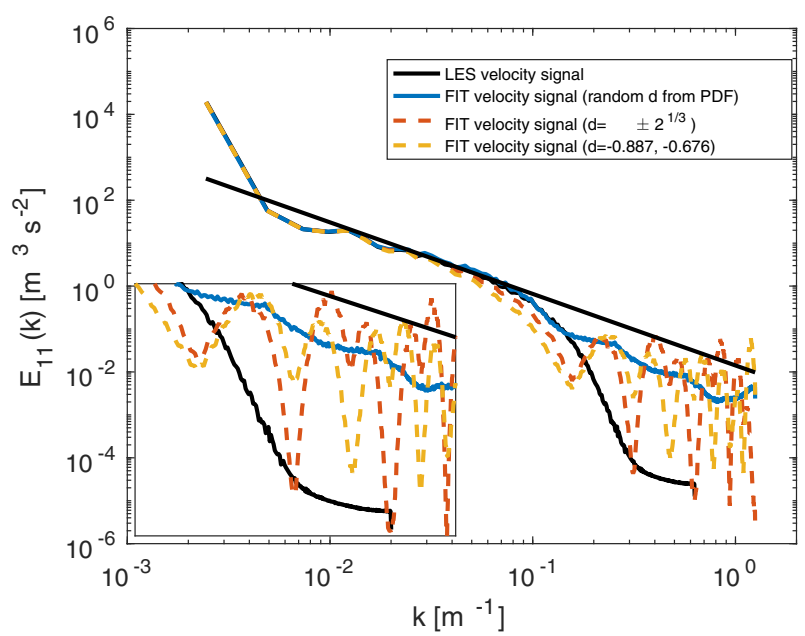

Fig. 20 Longitudinal energy spectra of LES velocity field and FIT-reconstructed field with a constant stretching parameter $d= \pm 2^{-1 / 3}, d=-0.88,-0.676$ and random stretching parameters from PDF

Figure 21 presents the PDFs of velocity increments for $u, v$ and $w$ components, separately, calculated at the distance $r=5 \mathrm{~m}$ at horizontal plane $z=595 \mathrm{~m}$. We compare LES (without filtering) with the FIT-reconstructed field. As it is seen, LES results are closer to Gaussian, while PDFs calculated from the FIT-reconstructed field have the exponential tails. This could be explained by the fact that the smallest resolved scales of LES are spuriously damped due to numerical diffusion, hence, the inertial-range intermittency is not reproduced by LES velocity field.

As reported by [20], a limitation of the fractal model is that the sub-grid scale velocity field is not divergence free due to the loss of the correlation between directions. To investigate this issue, the error in mass conservation at different reconstruction steps is calculated. For this, the following formula is used

$$
\delta_{\nabla \mathbf{u}}=\sqrt{\frac{1}{n \cdot m \cdot p} \sum_{i=1}^{n} \sum_{j=1}^{m} \sum_{k=1}^{p}(\nabla \cdot \vec{u})_{i j k}^{2}} .
$$

where $n, m, p$ is the number of grid points in $x, y$ and $z$ directions, respectively. The divergence of velocity was calculated with the central difference scheme. Additionally, the error in mass conservation is characterized by the quantity $|\max (\nabla \cdot \vec{u})-\min (\nabla \cdot \vec{u})|$. LES field was filtered to $k_{c u t}=0.150,0.075$ or $0.037 \mathrm{~m}^{-1}$, which corresponds to the grid resolution of approximately 10, 20 and $40 \mathrm{~m}$, respectively. Next, the signal was reconstructed back to the resolution $5 m$, which means that 1 FIT reconstruction step was applied to the signal with $k_{\text {cut }}=0.150 \mathrm{~m}^{-1}, 2$ steps to the signal with $k_{\text {cut }}=0.075 \mathrm{~m}^{-1}$ and 3 steps to the signal with $0.037 m^{-1}$. Results are presented in Table 1 .

In theory, $\delta_{\nabla \mathbf{u}}$ should be zero but due to numerical errors, the divergence of LES velocity fields are usually very small (not zero). We first note that, as seen in Table 1, there is a difference in $\delta_{\nabla \mathbf{u}}$ of two orders of magnitude between LES and the filtered LES without FIT reconstruction. In spite of this, filtered velocity fields are commonly used to do a priori tests to estimate e.g. an effect of SGS models on particle statistics [14, 29, 64]. Each FIT reconstruction step increases the error in mass conservation, however, after two steps $\delta_{\nabla \mathbf{u}}$ is 
Fig. 21 a PDFs of LES and FIT velocity increments $\delta u$, b PDFs of LES and FIT velocity increments $\delta v$, (c) PDFs of LES and FIT velocity increments $\delta w$. FIT with random $d$ was applied
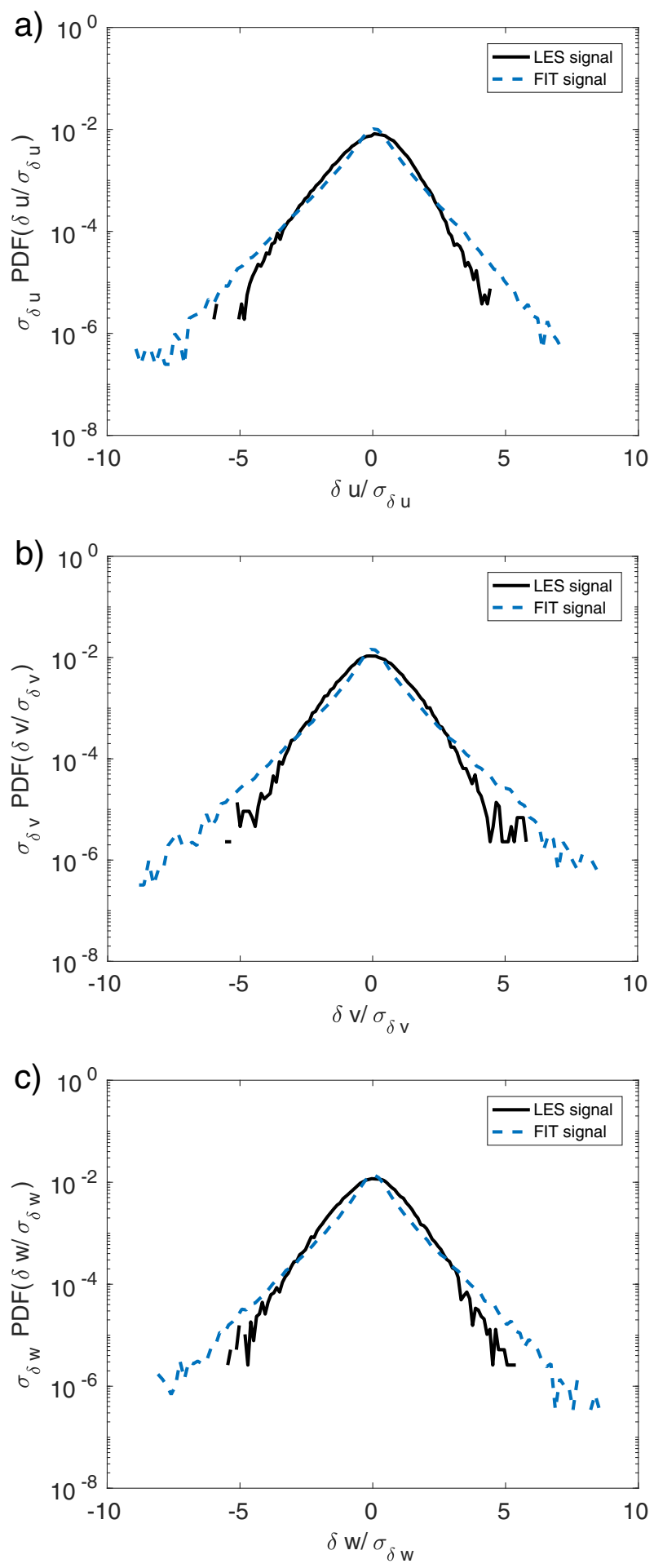
Table 1 Values of $\delta_{\nabla \mathbf{u}}$ for different $k_{c u t}$ and different fields

\begin{tabular}{lllll}
\hline Method & $k_{c u t}\left[\mathrm{~m}^{-1}\right]$ & $\begin{array}{l}\text { Number of } \\
\text { reconstruction } \\
\text { steps }\end{array}$ & $\delta_{\nabla \mathbf{u}}$ & \\
& & $\max (\nabla \cdot \vec{u})-\min (\nabla \cdot \vec{u}) \mid$ \\
\hline LES - no filtering & 0.3 & 0 & 0.00003 & 0.00051 \\
Filtered LES - no FIT & 0.037 & 0 & 0.0036 & 0.0554 \\
FIT with constant & 0.150 & 1 & 0.0106 & 0.2023 \\
$d= \pm 2-1 / 3$ & 0.075 & 2 & 0.0142 & 0.4379 \\
& 0.037 & 3 & 0.0218 & 0.7189 \\
FIT with constant & 0.150 & 1 & 0.0066 & 0.1395 \\
$d=-0.887,-0.676$ & 0.075 & 2 & 0.0097 & 0.2849 \\
& 0.037 & 3 & 0.0156 & 0.6322 \\
FIT with random & 0.150 & 1 & 0.0064 & 0.1253 \\
values of $d$ & 0.075 & 2 & 0.0087 & 0.2962 \\
from PDF & 0.037 & 3 & 0.0105 & 0.6052 \\
\hline
\end{tabular}

still of the same order of magnitude as the error of filtered LES without reconstruction, at least for the FIT method with random $d$ and for FIT with constant $d=-0.887,-0.676$.

The difference between the maximum and minimum of the divergence of the velocity fields is seen to be one order of magnitude larger if all FIT models are compared with filtered LES. Of all FIT approaches, the new proposal with random values of $d$ gives a smallest value of $\delta_{\nabla \mathbf{u}}$ and $|\max (\nabla \cdot \vec{u})-\min (\nabla \cdot \vec{u})|$. Based on this result, we suggest that one or two iteration steps of FIT to LES velocity field should not be exceeded to keep the error in mass conservation at an acceptable level.

Next, we investigated computational cost of the FIT procedure applied to three velocity components in $3 D$ space. We perform one to four reconstruction steps. As presented in Fig. 22, the computational cost (CPU time) of one, two or even three FIT reconstruction

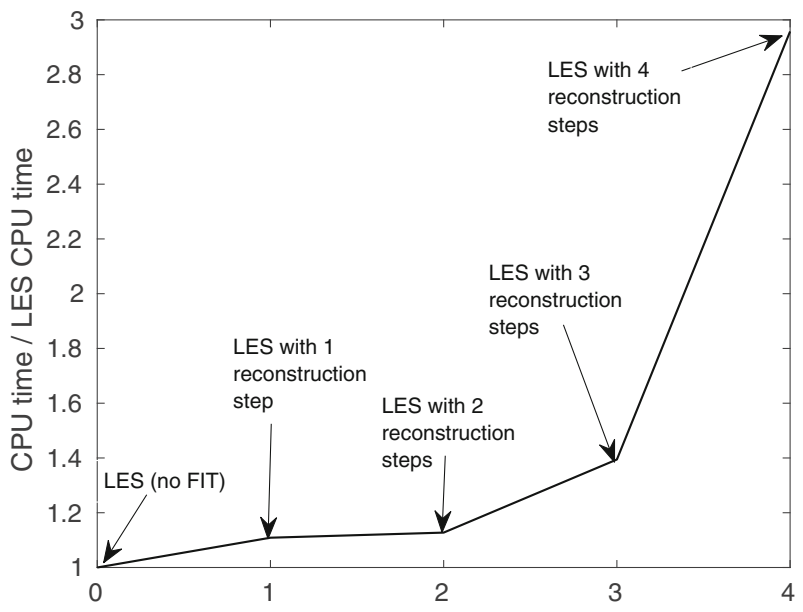

Fig. 22 Additional computational time required for fractal interpolation technique 
steps is small compared to the time needed for LES to resolve large scale features. This shows that the newly proposed fractal model is not only able to reproduce inertial-range eddies but is also computationally efficient.

\section{Conclusions}

In this work, a fractal model for the reconstruction of subgrid scales in large eddy simulation is presented. For the reconstruction process, values of the stretching parameter $d$ should be specified. This parameter determines the characteristics of the reconstructed signal which can be derived from its fractal dimension [20]. In previous works, the stretching parameter was chosen to be constant in time and space. To account for its spatial variability, we estimate the PDF of the absolute value of the stretching parameter $|d|$ from DNS data of stratocumulus-top boundary layer, LES data in the same flow configuration and, additionally, measurement data from POST campaign. For this, 1D intersections of velocity field are first low-pass filtered to certain cut-off wavenmbers. Next, an algorithm proposed by [34] is used to compute the local values of $d$. It was found that if the cut-off wavenumbers were in the inertial range, the PDFs of the stretching parameter collapsed into one curve, independent of the Reynolds number.

Next, 1D intersections of filtered velocity field were reconstructed such that $d$ is a random variable with the prescribed, previously determined PDF. Performance of the new approach was compared with FITs with constant values of $d$. It was shown the energy spectra follow the $-5 / 3$ scaling more closely and have no spurious modulations if $d$ is random. Moreover, the non-Gaussian, stretched-exponential tails of PDFs of velocity increments are reproduced corretly by the improved model. We investigated these statistics as they quantify internal intermittency of small scale turbulence [32, 41].

The random stretching parameter is also used to construct unresolved scales of POST airborne data (flight 13) and 3-D LES of stratocumulus-top boundary layer. Statistics of velocity increments showed that the improved fractal sub-grid model is capable to reproduce some of the sub-grid scale features, which might be lost due to finite grid resolution and numerical effects in LES or finite sampling frequency of measurements.

In the case of LES filed, the fractal reconstruction was extended to three dimensions. The divergence-free condition, which can be violated when using fractal interpolation technique, was addressed. We observe that after two reconstruction steps, the error in mass conservation of the reconstructed field is of the same order of magnitude as the error of filtered LES without reconstruction. Moreover, the computational cost required by FIT was compared with the cost of LES without reconstruction. We conclude that, even with three reconstruction steps, CPU time of LES with FIT is of the same order of magnitude as the CPU time of LES.

A perspective for a further study is to use this fractal model to reconstruct sub-grid scales of a carrier fluid flow and study the motion of Lagrangian particles in atmospheric physics applications, see e.g. [65-67].

Acknowledgements This work received funding from the European Union Horizon 2020 Research and Innovation Programme under the Marie Sklodowska-Curie Actions, Grant Agreement No. 675675.

MW and SPM acknowledge matching fund from the Polish Ministry of Science and Higher Education No. 341832/PnH/2016. The authors acknowledge Z. Wacławczyk for helping with Fig. 3. 
Open Access This article is distributed under the terms of the Creative Commons Attribution 4.0 International License (http://creativecommons.org/licenses/by/4.0/), which permits unrestricted use, distribution, and reproduction in any medium, provided you give appropriate credit to the original author(s) and the source, provide a link to the Creative Commons license, and indicate if changes were made.

\section{References}

1. Fox, R.O.: Large Eddy simulation tools for multiphase flows. Ann. Rev. Fluid Mech. 44, 47-76 (2012)

2. Marchioli, C., Salvetti, M.V., Soldati, A.: Appraisal of energy recovering sub-grid scale models for large eddy simulation of turbulent dispersed flows. Acta Mech 201, 277-296 (2008)

3. Marchioli, C.: Large-eddy simulation of turbulent dispersed flows: a review of modelling approaches. Acta Mech 228, 741 (2017). https://doi.org/10.1007/s00707-017-1803-X

4. Armenio, V., Piomelli, U., Fiorotto, V.: Effect of the sub-grid scales on particle motion. Phys. Fluids 11, 3030 (1999)

5. Kuerten, J.G.M.: Point-particle DNS and LES of particle-laden turbulent flow - a state of the art review. Flow Turbul. Combust. 97, 689-713 (2016)

6. Minier, J.-P.: On Lagrangian stochastic methods for turbulent polydispersed two-phase reactive flows. Prog. Energy Combust Sci. 50, 1-62 (2015)

7. Pozorski, J., Wacławczyk, T., Łuniewski, M.: LES of turbulent channel flow and heavy particle dispersion. J. Theor. Appl. Mech. 45, 643-657 (2007)

8. Soldati, A.: Particles turbulence interactions in boundary layers. ZAMM -J. Applied Math. Mech. 85, 683-699 (2005)

9. Subramanian, S.: Lagrangian-eulerian methods for multiphase flows. Prog. Energy Combust Sci. 39, 215-245 (2013)

10. Yang, Y., He, G.W., Wang, L.P.: Effects of sub-grid scale modelling on Lagrangian statistics in large eddy simulation. J. Turbul. 9, N8 (2008)

11. Das, S.K., Durbin, P.A.: A Lagrangian stochastic model for dispersion in stratified turbulence. Phys. Fluids 17, 025109 (2005)

12. Innocenti, A., Marchioli, C., Chibbaro, S.: Lagrangian filtered density function for LES-based stochastic modelling of turbulent particle-laden flows. Phys. Fluids 28, 115106 (2016)

13. Pozorski, J., Wacławczyk, M., Minier, J.-P.: Full velocity-scalar probability density function computation of heated channel flow with wall function approach. Phys. Fluids 15, 1220-1232 (2003)

14. Pozorski, J., Apte, S.V.: Filtered particle tracking in isotropic turbulence and stochastic modeling of sub-grid scale dispersion. Int. J. Multiphase Flow 35, 118-128 (2009)

15. Borgas, M.S., Sawford, B.L.: A family of stochastic models for two-particle dispersion in isotropic homogeneous stationary turbulence. J. Fluid Mech. 279, 69-99 (1994)

16. Heppe, B.M.O.: Generalized Langevin equation for relative turbulent dispersion. J. Fluid Mech. 357, 167-198 (1998)

17. Cernick, M.J., Tullis, S.W., Lightstone, M.F.: Particle sub-grid scale modeling in large eddy simulations of particle-laden turbulence. J. Turbul. 16, 101-135 (2015)

18. Minier, J.-P., Pozorski, J.: Particles in wall-bounded turbulent flows: deposition, re-suspension and agglomeration. Springer 571, 0254-1971 (2017). https://doi.org/10.1007/978-3-319-41567-3

19. Barnley, M.: Fractal everywhere. Academy Press, Boston (1993)

20. Scotti, A., Meneveau, C.: A fractal model for large eddy simulation of turbulent flow. Physica D 127, 198-232 (1999)

21. Geurts, B.J.: Inverse modeling for large eddy simulation. Phys. Fluids 9, 3585 (1997)

22. Mellado, J.P., Sarkar, S.: Reconstruction subgrid models for nonpremixed combustion. Phys. Fluids 15, 3280 (2003)

23. Stolz, S., Adams, N.A., Kleiser, L.: An approximate deconvolution model for large eddy simulation with application to incompressible wall-bounded flows. Phys. Fluids 13, 997 (2001)

24. Zhou, Z., Wang, S., Jin, G.: A structural sub-grid scale model for relative dispersion in large eddy simulation of isotropic turbulent flows by coupling kinematic simulation with approximate deconvolution method. Phys. Fluids 30, 105110 (2018)

25. Gobert, C., Manhart, M.: Subgrid modeling for particle-LES by spectrally optimized interpolation (SOI). J. Comput. Phys. 230, 7796-7820 (2011)

26. Fung, J.C.H., Vassilicos, J.C.: Two-particle dispersion in turbulentlike flows. Phys.Rev.E 57, 1677-1690 (1998) 
27. Łuniewski, M., Pozorski, J.: Spatial distribution and settling velocity of heavy particles in synthetic turbulent fields. 5 Trans. Inst. Fluid-Flow Mach. 135, 87-100 (2017)

28. Malik, N.A., Vassilicos, J.C.: A Lagrangian model for turbulent dispersion with turbulent-like flow structure: comparison with direct numerical simulation for two-particle statistics. Phys.Fluids 11, 1572-1580 (1999)

29. Pozorski, J., Rosa, B.: The motion of settling particles in isotropic turbulence: filtering impact and kinematic simulations as subfilter model. In: Salvetti, M., Armenio, V., Fröhlich, J., Geurts, B., Kuerten, H. (eds.) Direct and Large-Eddy simulation XI. ERCOFTAC series, p. 25. Springer, Cham (2019)

30. Basu, S., Foufoula-Georgiou, E., Porte-Agel, F.: Synthetic turbulence, Fractal interpolation and Large Eddy simulation. Phys. Rev. E70, 026310 (2004)

31. Salvetti, M.V., Marchioli, C., Soldati, A.: Lagrangian tracking of particles in large eddy simulation with fractal interpolation, Conference on Turbulence and Interactions TI 2006 (2006)

32. Ishihara, T., Gotoh, T., Kaneda, Y.: Study of high-Reynolds number isotropic turbulence by direct numerical simulation. Annu. Rev. Fluid Mech. 41, 165-180 (2009)

33. Akinlabi, E.O., Wacławczyk, M., Malinowski S.P.: Fractal reconstruction of sub-grid scales for large eddy simulation of atmospheric turbulence. J. Phys.: Conf. Ser. 1101, 012001 (2018). https://doi.org/10. 1088/1742-6596/1101/1/0120012018

34. Mazel, D.S., Hayes, M.H.: Using iterated function systems to model discrete sequences. IEEE Trans. Signal Process 40(7), 1724-1734 (1992). https://doi.org/10.1109/78.143444

35. Mellado, J.P.: Cloud-top entrainment in stratocumulus clouds. Annu. Rev. Fluid Mech. 49, 145-169 (2017)

36. Pedersen, J.G., Ma, Y.-F., Grabowski, W.W., Malinowski, S.P.: Anisotropy of observed and simulated turbulence in marine stratocumulus. J. Adv. Model. Earth Syst. 10, 500-515 (2018)

37. Gerber, H., Frick, G., Malinowski, S.P., Jonsson, H., Khelif, D., Krueger, S.K.: Entrainment rates and microphysics in POST stratocumulus J. Geophys. Res. Atmos. 118. https://doi.org/10.1002/jgrd.50878, Guodong (2013)

38. Jen-La Plante, I., Ma, Y., Nurowska, K., Gerber, H., Khelif, D., Karpinska, K., Kopec, M.K., Kumala, W., Malinowski, S.P.: Physics of stratocumulus top (POST): turbulence characteristics. Atmos. Chem. Phys. 16(15), 9711-9725 (2016)

39. Malinowski, S.P., Gerber, H., Jen-La Plante, I., Kopeć, M.K., Kumala, W., Nurowska, K., Chuang, P.Y., Khelif, D., Haman, K.E.: Physics of stratocumulus top (POST): turbulent mixing across capping inversion. Atmos.Chem.and Phys. 13, 12171-12186 (2013)

40. Kamps, O., Friedrich, R., Grauer, R.: Exact relation between Eulerian and Lagrangian velocity increment statistics. Phys. Fluids E 79, 066301 (2009)

41. Lui, L., Hu, F., Cheng, X., Song, L.: Probability density functions of velocity increments in the atmospheric boundary layer. Boundary-Layer Meteorol. 134, 243-255 (2010)

42. Ayala, O., Rosa, B., Wang, L., Grabowski, W.W.: Effects of turbulence on the geometric collision rate of sedimenting droplets. Part 1. Results from direct numerical simulation. New J. Phys. 10, 075015 (2008)

43. Grabowski, W.W., Wang, L.: Growth of cloud droplets in a turbulent environment. Annu. Rev. Fluid Mech. 45, 293-324 (2013)

44. Mellado, J.P., Bretherton, C.S., Stevens, B., Wyant, M.C.: DNS and LES for simulating stratocumulus: better together. J. Adv. Model. Earth Syst. 10,1421-1438 (2018). https://doi.org/10.1029/2018MS001 312

45. Barnley, M.: Constr. Approx. 2, 303-329 (1986)

46. Orey, S.: Gaussian sample functions and the Hausdorf dimension of level crossings. Z. Wahrscheinlichkeitstheorie Verw Geb. 15, 249-256 (1970)

47. Praskovsky, A.A., Foss, J.F., Kleis, S.J., Karyakin, M.Y.: Fractal properties of isovelovity surfaces in high Reynolds number laboratory shear flows. Phys. Fluids A 5, 2038-2042 (1993)

48. Scotti, A., Meneveau, C., Saddoughi, S.G.: Fractal dimension of velocity signals in high Reynolds number hydrodynamic turbulence. Phys. Rev. E. 51, 5594-5608 (1995)

49. Matheou, G.: Turbulence structure in a stratocumulus cloud. Atmosphere 9(10), 392 (2018)

50. Schulz, B., Mellado, J.P.: Wind shear effects on radiatively and evaporatively driven stratocumulus tops. J. Atmos. Sci. 75, 3245-3263 (2018). https://doi.org/10.1175/JAS-D-18-0027.1

51. Stevens, B., Lenschow, D.H., Vali, G., Gerber, H., Bandy, A., Blomquist, B., Brenguier, J., Bretherton, C.S., Burnet, F., Campos, T., Chai, S., Faloona, I., Friesen, D., Haimov, S., Laursen, K., Lilly, D.K., Loehrer, S.M., Malinowski, S.P., Morley, B., Petters, M.D., Rogers, D.C., Russell, L., Savic-Jovcic, V., Snider, J.R., Straub, D., Szumowski, M.J., Takagi, H., Thornton, D.C., Tschudi, M., Twohy, C., Wetzel, M., van Zanten, M.C.: Dynamics and chemistry of marine stratocumulus-DYCOMS-II. Bull. Amer. Meteor. Soc. 84, 579-594 (2003). https://doi.org/10.1175/BAMS-84-5-579 
52. Faloona, I., Lenschow, D.H., Campos, T., Stevens, B., van Zanten, M., Bloomquist, B., Thorton, D., Bandy, A., Gerber, H.: Observations of entrainment in eastern pacific marine stratocumulus using three conserved scalars. J. Atmos.Sci. 62, 3268-3284 (2005)

53. Pope, S.B.: Turbulent flows. Cambridge University Press, Cambridge (2000)

54. Prusa, J.M., Smolarkiewicz, P.K., Wyszogrodzki, A.A.: EULAG, a computational model for multiphase flows. Int. J. Comput. Fluids 37, 1193-1207 (2008). https://doi.org/10.1016/j.compfluid.2007.12.001

55. Piotrowski, Z.P., Smolarkiewicz, P.K., Malinowski, S.P., Wyszogrodzki, A.A.: On numerical realizability of thermal convection. J. Comp. Physics 228, 6268-6290 (2009). https://doi.org/10.1016/j.jcp.2009.05. 023

56. Kurowski, M.J., Malinowski, S.P., Grabowski, W.W.: A numerical investigation of entrainment and transport within a stratocumulus-topped boundary layer. Q. J. R. Meteorol. Soc. 135, 77-92 (2009)

57. Stevens, B., Moeng, C.-H., Ackerman, A.S., Bretherton, C.S., Chlond, A., de Roode, S., et al.: Evaluation of large-eddy simulations via observations of nocturnal marine stratocumulus. Mon. Weather. Rev. 133, 1443-1462 (2005). https://doi.org/10.1175/MWR2930.1

58. Smolarkiewicz, P.K.: Multidimensional positive definite advection transport algorithm: an overview. Int. J. Numer. Meth. Fluids 50, 1123-1144 (2006)

59. Weinstein, C.J.: IEEE acoustics, speech, and signal processing society: digital signal processing committee programs for digital signal processing. IEEE Press, New York (1979)

60. Akinlabi, E.O., Wacławczyk, M., Mellado, J.-P., Malinowski, S.P.: Estimating turbulence kinetic energy dissipation rates in the numerically simulated stratocumulus cloud-top mixing layer: evaluation of different methods. J. Atmos. Sci. 76, 1471-1488 (2019). https://doi.org/10.1175/JAS-D-18-0146.1

61. Devroye, L.: Non-uniform random variate generation. Springer, New York (1986)

62. Kailasnath, P., Sreenivasan, K.R., Stolovitzky, G.: Probability density of velocity increments in turbulent flows. Phys. Rev. Lett. 68, 2766 (1992)

63. Siebert, H., Lehmann, K., Wendisch, M.: Observations of small-scale turbulence and energy dissipation rates in the cloudy boundary layer. J. Atmos. Sci. 63, 1451-1466 (2006)

64. Guodong, J., Guo-Wei, H., Lian-Ping, W.: Large-eddy simulation of turbulent collision of heavy particles in isotropic turbulence. Phys. Fluids 22(5), 055106 (2010). https://doi.org/10.1063/1.3425627

65. Arabas, S., Shima, S.: Large-Eddy simulations of trade wind cumuli using particle-based microphysics with monte carlo coalescence. J. Atmos. Sci. 70, 2768-2777 (2013). https://doi.org/10.1175/JAS-D-120295.1

66. Dziekan, P., Pawlowska, H.: Stochastic coalescence in Lagrangian cloud microphysics. Atmos. Chem. Phys. 17, 13509-13520 (2017). https://doi.org/10.5194/acp-17-13509-2017

67. Grabowski, W.W., Abade, G.C.: Broadening of cloud droplet spectra through eddy hopping: turbulent adiabatic parcel simulations. J. Atmos. Sci. 74, 1485-1493 (2017). https://doi.org/10.1175/JAS-D-170043.1

68. Beghein, C., Allery, C., Wacławczyk, M., Pozorski, J.: Application of POD-based dynamical systems to dispersion and deposition of particles in turbulent channel flow. Int. J. Multiphase Flow 58, 97-113 (2014). https://doi.org/10.1016/j.ijmultiphaseflow.2013.09.001

Publisher's Note Springer Nature remains neutral with regard to jurisdictional claims in published maps and institutional affiliations. 